TRANSACTIONS OF THE

AMERICAN MATHEMATICAL SOCIETY

Volume 301, Number 2, June 1987

\title{
LATTICE EMBEDDINGS IN THE RECURSIVELY ENUMERABLE TRUTH TABLE DEGREES
}

\author{
CHRISTINE ANN HAUGHT
}

\begin{abstract}
It is shown that every finite lattice, and in fact every recursively presentable lattice, can be embedded in the r.e. $\mathrm{tt}$-degrees by a map preserving least and greatest elements. The decidability of the 1-quantifier theory of the r.e. $\mathrm{tt}$ degrees in the language with $\leqslant, \vee, \wedge, 0$, and 1 is obtained as a corollary.
\end{abstract}

Introduction. A set $A \subseteq \omega$ is truth table (tt) reducible to $B \subseteq \omega\left(A \leqslant{ }_{\mathrm{tt}} B\right)$ if answers to questions of the form " $n \in A$ ?" are given by a finite Boolean combination, effectively determined from $n$, of answers to questions of the form " $k \in B$ ?". Sets $A$ and $B$ are of the same tt-degree if $A \leqslant{ }_{\mathrm{tt}} B$ and $B \leqslant_{\mathrm{tt}} A$. We consider the structure consisting of the tt-degrees of recursively enumerable sets of natural numbers. Odifreddi [4] and Rogers [6] contain background information on the tt-degrees. In particular, Fejer and Shore [1] contains information about the r.e. tt-degrees and about questions relating to the decidability of the theory of the r.e. tt-degrees. They show there that every recursively presentable lattice can be embedded in the r.e. $\mathrm{tt}$-degrees preserving least element. Using this, they show that the $\exists$ theory of the r.e. $t$-degrees in the language with $\leqslant, \vee, \wedge, 0$ is decidable, and ask whether the $\exists$ theory is still decidable when 1 is added to the language. This decidability question can be answered by determining which finite lattices can be embedded in the r.e. $t$-degrees preserving least and greatest elements. Jockusch and Mohrherr [3] have shown that the diamond lattice, the pentagon lattice, and the 1-n-1 lattices can be embedded preserving least and greatest elements, but leave open the general question, and even such special cases as the three generator Boolean algebra. The embedding used in their proof requires that the lattice in question have the property that no element which is the inf of a pair of incomparable elements of the lattice can be joined up to the 1 of the lattice (except by 1 itself). We show here that all finite lattices, and in fact all recursively presentable lattices, can be embedded in the r.e. tt-degrees preserving least and greatest elements (provided the lattice has distinct least and greatest elements). Our proof for the general lattices combines a generalization of the coding method used by Jockusch and Mohrherr with the strategy for preserving nonzero infs used by Fejer and Shore.

We prove first, in $\S 1$, that every lattice with a finite representation (and distinct least and greatest elements) can be embedded, and then in $\$ 2$ outline the modifications needed to embed a recursively presented (possibly infinite) lattice. As a

Received by the editors December 26, 1985 and, in revised form, May 15, 1986.

1980 Mathematics Subject Classification (1985 Revision). Primary 03D30; Secondary 03D25.

(C)1987 American Mathematical Society $0002-9947 / 87 \$ 1.00+\$ .25$ per page 
corollary to this, we then show that the existential theory of the r.e. $t$-degrees in the language with $\leqslant, \vee, \wedge, 0,1$ is decidable.

Our notation is standard and follows that of Fejer and Shore [1] very closely. We make implicit use of the convention that if $\{e\}_{s}(x) \downarrow$, then $x<s$. The material in this paper appears as part of my doctoral dissertation [2]. I am grateful to Richard Shore for his guidance.

1. Finite lattices. We first explain our notation and outline some of the ideas which will be used throughout the proof. The organization of the proof follows that of Fejer and Shore [1].

If $\{e\}(x) \downarrow$, then $[e](x)$ is the truth table with index $\{e\}(x)$, and $|[e](x)|$ is the size of the truth table. If $A$ is a set of natural numbers, then we define $[e]^{A}$ by

$$
[e]^{A}(x)= \begin{cases}1 & \text { if }\{e\}(x) \downarrow \text { and } A \text { satisfies }[e](x), \\ 0 & \text { if }\{e\}(x) \downarrow \text { and } A \text { does not satisfy }[e](x), \\ \uparrow & \text { if }\{e\}(x) \uparrow,\end{cases}
$$

and $[e]_{s}^{A}$, by

$$
[e]_{s}^{A}(x)= \begin{cases}{[e]^{A}(x)} & \text { if }\{e\}_{s}(x) \downarrow, \\ \uparrow & \text { if }\{e\}_{s}(x) \uparrow .\end{cases}
$$

Sometimes we write $[e]^{A_{s}}$ for $[e]_{s}^{A_{s}}$, to simplify notation. So $A \leqslant{ }_{\mathrm{tt}} B$ iff for some $e$, $A=[e]^{B}$.

Let $\mathscr{P}=\left\langle P, \leqslant, \vee, \wedge, 0_{\mathscr{P}}, 1_{\mathscr{P}}\right\rangle$ be a finite lattice with $P=\left\{p_{i}: i \in[0, n]\right\}$ and $p_{0}=0_{\mathscr{P}}, p_{n}=1_{\mathscr{P}}$. A representation of $\mathscr{P}$ will be a set $\Phi=\left\{\alpha_{r}: r \in J\right\}$, where for $r \in J, \alpha_{r}:[0, n] \rightarrow \omega$, and for all $i, j, k \leqslant n$ the following hold:

$$
\begin{gathered}
p_{i} \leqslant p_{j} \leftrightarrow\left(\forall r_{1}, r_{2} \in J\right)\left[\alpha_{r_{1}}(j)=\alpha_{r_{2}}(j) \rightarrow \alpha_{r_{1}}(i)=\alpha_{r_{2}}(i)\right], \\
p_{i} \vee p_{j}=p_{k} \leftrightarrow\left(\forall r_{1}, r_{2} \in J\right) \\
{\left[\alpha_{r_{1}}(i)=\alpha_{r_{2}}(i) \text { and } \alpha_{r_{1}}(j)=\alpha_{r_{2}}(j) \rightarrow \alpha_{r_{1}}(k)=\alpha_{r_{2}}(k)\right],} \\
p_{i} \wedge p_{j}=p_{k} \leftrightarrow\left(\forall r_{1}, r_{2} \in J\right)\left[\alpha_{r_{1}}(k)=\alpha_{r_{2}}(k) \rightarrow \exists m \in \omega\right. \\
\exists s_{0}, \ldots, s_{m} \in J\left(s_{0}=r_{1} \text { and } s_{m}=r_{2} \text { and for } h<m-1, h\right. \text { even, } \\
\left.\left.\alpha_{s_{h}}(i)=\alpha_{s_{h+1}}(i) \text { and } \alpha_{s_{h+1}}(j)=\alpha_{s_{h+2}}(j)\right)\right] .
\end{gathered}
$$

For example, consider the lattice

$$
\mathscr{P}=\left(\left\{p_{0}, p_{1}, p_{2}, p_{3}, p_{4}, p_{5}\right\}, \leqslant, \vee, \wedge, 0_{\mathscr{P}}, 1_{\mathscr{P}}\right)
$$

with $0_{\mathscr{P}}=p_{0}, 1_{\mathscr{P}}=p_{5}$ and the ordering, join and meet given by the following diagram. Notice that $\mathscr{P}$ is a simple example of a lattice which cannot be embedded using Jockusch and Mohrherr's proof [3]. An example of a representation for $\mathscr{P}$ is the set of rows $\Phi=\left\{\alpha_{r}: r \in[0,6]\right\}$, where $\alpha_{r}:[0,5] \rightarrow[0,6]$, and the values for $\alpha_{r}(i)$ for $r \in[0,6]$ and $i \in[0,5]$ are given by Table 1 . 


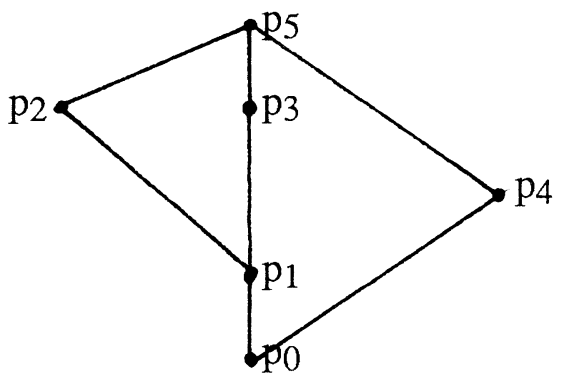

Rows $0,1,2$, and 3 establish (1) for all of the relationships $p_{i} \nless p_{j}$ which hold in $\mathscr{P}$, and rows 4,5 , and 6 give the interpolants whose existence is asserted by (3). The table maintains (1) and (2) for all of the relationships $p_{i} \leqslant p_{j}$ and $p_{i} \vee p_{j}=p_{k}$ which hold in $\mathscr{P}$.

\begin{tabular}{|c|c|c|c|c|c|c|}
\hline & & & LE 1 & & & \\
\hline & 0 & 1 & 2 & 3 & 4 & $\underline{5}$ \\
\hline$\underline{0}$ & 0 & 0 & 0 & 0 & 0 & 0 \\
\hline 1 & 0 & 1 & 1 & 1 & 0 & 1 \\
\hline$\underline{2}$ & 0 & 1 & 2 & 1 & 2 & 2 \\
\hline 3 & 0 & 1 & 2 & 3 & 3 & 3 \\
\hline 4 & 0 & 4 & 4 & 4 & 3 & 4 \\
\hline$\underline{5}$ & 0 & 5 & 5 & 4 & 0 & 5 \\
\hline 6 & 0 & 0 & 0 & 6 & 2 & 6 \\
\hline
\end{tabular}

A representation is called finite if $J$ (the set of indices for the rows) is finite. Pudlak and Tuma [5] have shown that every finite lattice has a finite representation. In this section we consider only finite lattices, so we use their result and assume that we have a finite representation for the lattice we embed. This will simplify the construction of the embedding somewhat. In $\$ 2$ we will prove that every lattice with a recursive presentation can be embedded preserving least and greatest elements. It is easy to see that every finite lattice has a recursive presentation. It will then not be necessary to use the difficult result of Pudlak and Tuma. So we assume, without loss of generality, that for some $d \in \omega, J=[0, d], n>0$, and for $r_{1}, r_{2} \leqslant d$, $\alpha_{r_{1}}:[0, n] \rightarrow[0, d], \alpha_{r_{1}}(0)=0$, and $\left(r_{1} \neq r_{2} \rightarrow \alpha_{r_{1}}(n) \neq \alpha_{r_{2}}(n)\right)$.

As in Fejer and Shore [1], our construction will define a recursive function, $f(x, s)$, where $f: \omega \times \omega \rightarrow[0, d]$. We define r.e. sets, $A_{i}, i \leqslant n$ by

$$
A_{i, s}=\left\{\left\langle x, \alpha_{f\left(x, s^{\prime}\right)}(i)\right\rangle: x \in \omega \text { and } s^{\prime} \leqslant s\right\}
$$

and

$$
A_{i}=\left\{\left\langle x, \alpha_{f(x, s)}(i)\right\rangle: x \in \omega \text { and } s \in \omega\right\} .
$$

The map $p_{i} \rightarrow \operatorname{deg}_{\mathrm{tt}}\left(A_{i}\right)$ will provide the embedding of $\mathscr{P}$ into the r.e. tt-degrees. 
Our construction will ensure the following:

$$
\begin{gathered}
A_{0} \text { is recursive. } \\
p_{i} \leqslant p_{j} \rightarrow A_{i} \leqslant{ }_{\mathrm{tt}} A_{j} . \\
p_{i} \vee p_{j}=p_{k} \rightarrow A_{k} \leqslant{ }_{\mathrm{tt}} A_{i} \oplus A_{j} . \\
K \leqslant{ }_{\mathrm{tt}} A_{n}, \text { where } K \text { is a fixed complete r.e. set. } \\
p_{i} \nless p_{j} \rightarrow A_{i} \star_{\mathrm{tt}} A_{j} . \\
p_{i} \nless p_{j} \text { and } p_{j} \nless p_{i} \text { and } p_{i} \wedge p_{j}=p_{k} \rightarrow \text { if }\left(Y \leqslant{ }_{\mathrm{tt}} A_{i}\right. \text { and } \\
\left.Y \leqslant{ }_{\mathrm{tt}} A_{j}\right) \text { then } Y \leqslant{ }_{\mathrm{tt}} A_{k} .
\end{gathered}
$$

Our definition of $A_{0}$ will guarantee that (4) holds, since for all $r \leqslant d, \alpha_{r}(0)=0$, so $A_{0}=\left\{\left\langle x, \alpha_{r}(0)\right\rangle: x \in \omega, r \in J\right\}=\{\langle x, 0\rangle: x \in \omega\}$ is recursive. The definition of the $A_{i}$ will also guarantee that (5) holds. Suppose $p_{i} \leqslant p_{j}$. Then

$$
\begin{aligned}
\langle x, y\rangle \in A_{i} & \leftrightarrow \exists s\left(\alpha_{f(x, s)}(i)=y\right) \\
& \leftrightarrow \exists r \leqslant d\left(\left\langle x, \alpha_{r}(j)\right\rangle \in A_{j} \text { and } \alpha_{r}(i)=y\right) .
\end{aligned}
$$

Condition (6) will be established by a simple restriction on the function $f$. We insist that

(10) For each $x$ there is at most one $s$ such that $f(x, s+1) \neq f(x, s)$.

Then if $p_{i} \vee p_{j}=p_{k}$, we get $A_{k} \leqslant{ }_{\mathrm{tt}} A_{i} \oplus A_{j}$ as follows:

$$
\begin{aligned}
\langle x, y\rangle \in A_{k} \leftrightarrow y & =\alpha_{f(x, 0)}(k) \\
& \text { or }\left(y \neq \alpha_{f(x, 0)}(k) \text { and } \exists s>0 y=\alpha_{f(x, s)}(k)\right) .
\end{aligned}
$$

Condition (2) allows us to recognize the right side of (11) in a tt-way using $A_{i} \oplus A_{j}$. If $\alpha_{f(x, 0)}(k) \neq \alpha_{f(x, s)}(k)$, then by (2), either $\alpha_{f(x, 0)}(i) \neq \alpha_{f(x, s)}(i)$ or $\alpha_{f(x, 0)}(j) \neq$ $\alpha_{f(x, s)}(j)$. Hence from (11) we get

$$
\begin{aligned}
\langle x, y\rangle \in A_{k} \leftrightarrow y=\alpha_{f(x, 0)}(k) \\
\text { or } \exists r \leqslant d\left[y=\alpha_{r}(k) \text { and }\left\langle x, \alpha_{r}(i)\right\rangle \in A_{i}\right. \\
\text { and }\left\langle x, \alpha_{r}(j)\right\rangle \in A_{j} \text { and } \forall z \leqslant d \\
\\
\left(\left(\langle x, z\rangle \in A_{i} \rightarrow z=\alpha_{r}(i) \text { or } z=\alpha_{f(x, 0)}(i)\right)\right. \\
\left.\left.\quad \text { and }\left(\langle x, z\rangle \in A_{j} \rightarrow z=\alpha_{r}(j) \text { or } z=\alpha_{f(x, 0)}(j)\right)\right)\right]
\end{aligned}
$$

and this provides the desired tt-reduction from $A_{i} \oplus A_{j}$.

The construction will guarantee that (7), (8) and (9) hold. Before describing this in detail, we need more notation. Let $h \in \omega$ be such that there are $h$ many distinct pairs, $\left(p_{i}, p_{j}\right)$ in $\mathscr{P}$ such that $p_{i} \nless p_{j}$. Let $\left\{“ p_{i_{a}} \nless p_{j_{a}}\right.$ ”: $\left.1 \leqslant a \leqslant h\right\}$ be a listing of all such relations. To establish (8) we will satisfy the requirements.

$$
P_{e}^{a}: A_{i_{a}} \neq[e]^{A_{j_{a}}}, \quad \text { for } 1 \leqslant a \leqslant h \text { and } e \in \omega .
$$


Let $m \in \omega$ be such that there are $(m-h)$ many distinct triples, $\left(p_{i}, p_{j}, p_{k}\right)$, in $\mathscr{P}$ such that $p_{i} \nless p_{j}$ and $p_{j} \nless p_{i}$ and $p_{i} \wedge p_{j}=p_{k}$. Let $\left\{“ p_{i_{a}} \wedge p_{j_{a}}=p_{k_{a}} ": h+1 \leqslant a\right.$ $\leqslant m\}$ be a listing of all such relations. To establish (9) we will satisfy the requirements

$$
Q_{e}^{a}:[e]^{A_{i_{a}}}=[e]^{A_{j_{a}}}=Y \rightarrow Y \leqslant{ }_{\mathrm{tt}} A_{k_{a}} \text { for } h+1 \leqslant a \leqslant m \text { and } e \in \omega .
$$

Next we recursively partition $\omega$ and associate elements of the partition so that

(15) Each $P_{e}^{a}$ has an infinite set of numbers associated with it. Let $D(\langle e, a\rangle)$ be the set of numbers associated with $P_{e}^{a}$.

(16) Each $x \in \omega$ has two finite sets of numbers associated with it, call them $W(x)$ and $C W(x)$. Each element of $W(x) \cup C W(x)$ is greater than $x$, and $\min (C W(x))$ $>\max (W(x))$ and

$$
\begin{aligned}
& |W(x)|=2^{x+1}(h+2(m-h)) \text { and } \\
& |C W(x)|=2(m-h)\left(2^{x+1}\right)(h+2(m-h)) .
\end{aligned}
$$

For each $x \in \omega$ we further partition $W(x)$ into $h+2(m-h)$ many pieces, each of size $2^{x+1}$, and label these so that

$$
W(x)=\bigcup_{a \in[1, h]} W^{a}(x) \cup\left(\bigcup_{(a, k) \in[h+1, m] \times\{1,2\}} W^{(a, k)}(x)\right),
$$

where for $a \in[1, h],\left|W^{a}(x)\right|=2^{x+1}$ and for $(a, k) \in[h+1, m] \times\{1,2\}$, $\left|W^{(a, k)}(x)\right|=2^{x+1}$. If $a \in[1, h]$ and $y \in W^{a}(x)$, we sometimes say that $y$ is an element of $W(x)$ "reserved for $a$ ", similarly for $(a, k) \in[h+1, m] \times\{1,2\}$.

We use the elements of $W(x)$ to witness $x \in K$. When we act to code $x$, our action will be chosen to cooperate with some requirement $P_{e}^{a}$ or $Q_{e}^{a}$. One witness will be used when we cooperate with $P_{e}^{a}$; two witnesses may be needed when we cooperate with $Q_{e}^{a}$. Since there are $h$ many different kinds of $P_{e}^{a}$ requirements, and $m-h$ many different kinds of $Q_{e}^{a}$ requirements, we have $h+2(m-h)$ many different sets of witnesses for coding $x$. Other requirements in the construction will be allowed to use up elements of our witness sets; we will use an inductive argument to show that $2^{x+1}$ elements for each requirement will be enough. The elements of $C W(x)$ serve as partners to the elements of $W(x)$, and are used to witness $x \notin K$. Each element of $W(x)$ will need two partners for each kind of inf requirement, hence we make $|C W(x)|=2(m-h)|W(x)|$. For each $x \in \omega$ and each triple $(y, a, k) \in W(x) \times[h+1, m] \times\{1,2\}$ we associate a unique element of $C W(x)$. We express this association via a recursive 1-1 function $g$ such that for each $x \in \omega$,

$$
g: W(x) \times[h+1, m] \times\{1,2\} \stackrel{\text { onto }}{\rightarrow} C W(x)
$$

and we call $g(y, a, k)$ a partner of $y$.

We now describe the strategies for satisfying each of (7), $P_{e}^{a}$ and $Q_{e}^{a}$ in isolation; after that we describe how these requirements interact and cooperate with each other. 
To get $K \leqslant_{\mathrm{tt}} A_{n}$, we establish

$$
\begin{aligned}
x \in K \leftrightarrow \exists y \in W(x)[ & \exists r \leqslant d\left(r \neq f(y, 0) \text { and }\left\langle y, \alpha_{r}(n)\right\rangle \in A_{n}\right) \\
& \text { and } \forall(q, a, k) \in[0, d] \times[h+1, m] \times\{1,2\} \\
& \left.\left(\left\langle g(y, a, k), \alpha_{q}(n)\right\rangle \in A_{n} \rightarrow q=f(g(y, a, k), 0)\right)\right] .
\end{aligned}
$$

Condition (17) says that $x$ is in $K$ if and only if there is a $y \in W(x)$ such that at some stage $s, f(y, s) \neq f(y, 0)$, but for all $z$ such that $z$ is a partner of $y$, and all stages $s^{\prime}, f\left(z, s^{\prime}\right)=f(z, 0)$. Let $\left\{k_{t}: t \in \omega\right\}$ be a 1-1 recursive enumeration of $K$, and $K_{s}=\left\{k_{t}: t<s\right\}$. During odd stages of the construction we will act for the sake of coding; during even stages of the construction we will act for the sake of other requirements. At stage $s+1=2 t+1$ we will try to establish (17) for $x=k_{t}$. We choose a $y \in W\left(k_{t}\right)$ and an $r \leqslant d$ such that $f(y, 2 t)=f(y, 0), r \neq f(y, 0)$, and for all $(a, k) \in[h+1, m] \times\{1,2\}, f(g(y, a, k), 2 t)=f(g(y, a, k), 0)$. The exact choice of $y$ and $r$ will depend on the interaction between coding and other requirements. We then set $f(y, 2 t+1)=r$ (thereby enumerating $\left\langle y, \alpha_{r}(n)\right\rangle$ into $\left.A_{n}\right)$ and insist that

$$
\forall s^{\prime} \geqslant 2 t+1 \forall z \in W\left(k_{t}\right) \cup C W\left(k_{t}\right)\left(f\left(z, s^{\prime}\right)=f(z,, 2 t+1)\right) .
$$

In this way, " $\rightarrow$ " of (17) is established.

At stage $s^{\prime}+1=2 t^{\prime}$ of the construction, it may be the case for some $x$ that $x \notin K_{s^{\prime}+1}$, but $\exists y \in W(x)\left(f\left(y, s^{\prime}+1\right) \neq f\left(y, s^{\prime}\right)\right)$. To maintain (17), for any such $y$ we insist that

$$
\begin{aligned}
& \exists(r, a, k) \in[0, d] \times[h+1, m] \times\{1,2\} \\
& \left(f\left(g(y, a, k), s^{\prime}+1\right)=r \text { and } r \neq f(g(y, a, k), 0)\right) .
\end{aligned}
$$

The schemes for cooperation among the requirements will guarantee that each of these actions is possible.

We will use numbers in the set $D(\langle e, a\rangle)$ to satisfy the requirements $P_{e}^{a}$, for $a \in[1, h], e \in \omega$. Since $p_{i_{a}} \nless p_{j_{a}}$, by (1) we get that there are $r(a)_{1}, r(a)_{2} \leqslant d$ such that for $a \in[1, h]$

$$
\alpha_{r(a)_{1}}\left(j_{a}\right)=\alpha_{r(a)_{2}}\left(j_{a}\right) \text { and } \quad \alpha_{r(a)_{1}}\left(i_{a}\right) \neq \alpha_{r(a)_{2}}\left(i_{a}\right) .
$$

For each $y \in D(\langle e, a\rangle)$ we set $f(y, 0)=r(a)_{1}$. At stage $s+1=2 t$ during the construction, if there is an $x \in D(\langle e, a\rangle)$ such that $f(x, s)=f(x, 0)=r(a)_{1}$ and

$$
[e]^{A_{j_{a} \cdot s}}\left(\left\langle x, \alpha_{r(a)_{2}}\left(i_{a}\right)\right\rangle\right) \downarrow=0,
$$

then $P_{e}^{a}$ tries to set $f(x, s+1)=r(a)_{2}$, and to preserve $A_{j_{a}, s} \uparrow\left|[e]\left(\left\langle x, \alpha_{r(a)_{2}}\left(i_{a}\right)\right\rangle\right)\right|$. In this way we enumerate $\left\langle x, \alpha_{r(a)_{2}}\left(i_{a}\right)\right\rangle$ into $A_{i_{a}, s+1}$ and cause

$$
A_{i_{a}, s+1}\left(\left\langle x, \alpha_{r(a)_{2}}\left(i_{a}\right)\right\rangle\right) \neq[e]^{\mathcal{A}_{j_{a} s+1}}\left(\left\langle x, \alpha_{r(a)_{2}}\left(i_{a}\right)\right\rangle\right) .
$$


Next, we consider a requirement $Q_{e}^{a}$, for $a \in[h+1, m]$ and $e \in \omega$. We try to make $[e]^{A_{i_{a}}} \neq[e]^{A_{j_{a}}}$; if this fails then we must be able to recover $Y=[e]^{A_{i_{a}}}=[e]^{A_{j_{a}}}$ from $A_{k_{a}}$ in a tt way. Suppose $s$ and $y$ are such that $\{e\}_{s}\left(y^{\prime}\right) \downarrow$ for all $y^{\prime} \leqslant y$. At stage $s$ there are two cases to consider. First, $[e]^{A_{i_{a}} \cdot s}\left(y^{\prime}\right) \neq[e]^{A_{j_{a} .}}\left(y^{\prime}\right)$ for some $y^{\prime} \leqslant y$, w.l.o.g. $y^{\prime}=y$. In this case we try to maintain the inequality on $y$ by either restraining all numbers $\leqslant|[e](y)|$ from $A_{i_{a}, s+1}$ and $A_{j_{a}, s+1}$ if possible, or by causing numbers to be enumerated into $A_{i_{a}, s+1}$ and $A_{j_{a}, s+1}$ or both in such a way that the inequality on $y$ remains. This method of enumerating is based on Jockusch and Mohrherr's method of preserving an inf in their embedding of the diamond lattice in the r.e. tt-degrees in [7] and will be described in more detail when we discuss the cooperation between coding and $Q_{e}^{a}$. The second case is $[e]^{A_{i_{a} . s}}\left(y^{\prime}\right)=$ $[e]^{A_{j_{a}, s}}\left(y^{\prime}\right)$ for all $y^{\prime} \leqslant y$. We would like to be able to either cause $[e]^{A_{i_{\alpha}, s+1}}(y) \neq$ $[e]^{A_{j_{a}, s+1}}(y)$, or to be able to assume that we can recover $[e]^{A_{j_{a}}}(y)$ from $A_{i_{a}, s}, A_{j_{a}, s}$ and $A_{k_{a}}$. To this end, we consider all numbers which may later be enumerated into $A_{i_{a}}$ or $A_{j_{a}}$ affecting the computations $[e]^{A_{i_{a} \cdot s}}(y)$ and $[e]^{A_{j_{a} s}}(y)$. If there is a way of enumerating some subset of these numbers into $A_{i_{a}, s+1} \cup A_{j_{a}, s+1}$ so that $[e]^{A_{i_{a}, s+1}}(y)$ $\neq[e]^{A_{j_{a} s+1}}(y)$, then we take this action. If this is impossible, then we will be able to argue, roughly, that all changes in $A_{i_{a}}$ after stage $s$ which affect the computation $[e]^{A_{i_{a}}}(y)$ occur because we defined $f$ in a way that also caused changes in $A_{j_{a}}$. The lattice representation will then cause all such changes to be reflected in $A_{k_{a}}$, and so we will be able to recover $[e]^{A_{i_{a}}}(y)$ from $A_{k_{a}}$.

Let $\left\{T_{i}: i \in \omega\right\}$ be an effective listing of the requirements $\left\{P_{e}^{a}: a \in[1, h], e \in \omega\right\}$ and $\left\{Q_{e}^{a}: a \in[h+1, m], e \in \omega\right\}$. We now describe the schemes for cooperation among the $T_{i}$ and the coding.

Coding and $T_{i}=P_{e}^{a}$. Since $D(\langle e, a\rangle)$ is disjoint from $\cup_{x \in \omega}(W(x) \cup C W(x))$, action taken for the sake of $T_{i}$ has no bearing on (17), and so has no interaction with the coding. Action taken for coding may interact with $P_{e}^{a}$, though. Suppose that $P_{e}^{a}$ has acted before stage $s+1=2 t+1$, and wants to preserve $A_{j_{a}} \uparrow u$. If $k_{t} \geqslant u$, then since all elements of $W\left(k_{t}\right)$ are greater than $k_{t}$, the action taken for coding will not disturb $P_{e}^{a}$. However, if $k_{t}<u$, then we would like to choose the coding action in such a way as to preserve $A_{j_{a}, s} \uparrow u$. Let $r(a)_{1}, r(a)_{2} \leqslant d$ be as defined in (19). We claim we can use $r(a)_{1}$ and $r(a)_{2}$ to enumerate a number into $A_{n, s+1}$ without disturbing $A_{j_{a}, s+1}$. For each $x \in \omega, a \in[1, h]$ and each $y \in W^{a}(x)$, we have set $f(y, 0)=r(a)_{1}$. Now if we decide that coding should cooperate with $P_{e}^{a}$ at $s+1=$ $2 t+1$, then we choose a $y \in W^{a}\left(k_{t}\right)$ such that $f(y, s)=f(y, 0)$ and for all $\left(a^{\prime}, k\right) \in[h+1, m] \times\{1,2\}, \quad f\left(g\left(y, a^{\prime}, k\right), s\right)=f\left(g\left(y, a^{\prime}, k\right), 0\right)$, and we set $f(y, s+1)=r(a)_{2}$. In this way we enumerate $\left\langle y, \alpha_{r(a)}(n)\right\rangle$ into $A_{n, s+1}$ and leave $A_{j_{a}, s+1}=A_{j_{a}, s}$. It will be necessary for coding to cooperate with $P_{e}^{a}$ at most finitely often, since there can only be finitely many $t$ with $k_{t}<u$.

Coding and $T_{i}=Q_{e}^{a}$. Action taken for coding may interact with $Q_{e}^{a}$. Suppose $s$ and $y$ are such that $\forall y^{\prime} \leqslant y\left(\{e\}\left(y^{\prime}\right) \downarrow\right)$ and $[e]^{A_{i_{a}, s}} \uparrow y=[e]^{A_{j_{a} .} \uparrow} \uparrow y$ and $[e]^{A_{i_{a} .}}(y)$ $\neq[e]^{A_{j_{a} s}}(y)$. If $s+1=2 t+1$ and $k_{t} \geqslant|[e](y)|$ then action taken for coding at $s+1$ will not disturb $Q_{e}^{a}$. However, if $k_{t}<|[e](y)|$, then we need to maintain the inequality at $y$. Since for all $a \in[h+1, m], p_{i_{a}} \neq 1_{\mathscr{P}}$ and $p_{j_{a}} \neq 1_{\mathscr{P}},(1)$ implies that 
there must be $r(a, 1)_{1}, r(a, 1)_{2}, r(a, 2)_{1}, r(a, 2)_{2} \in J$ satisfying

$$
\begin{aligned}
& \alpha_{r(a, 1)_{1}}\left(i_{a}\right)=\alpha_{r(a, 1)_{2}}\left(i_{a}\right) \quad \text { and } \\
& \alpha_{r(a, 1)_{1}}(n) \neq \alpha_{r(a, 1)_{2}}(n) \quad \text { and } \\
& \alpha_{r(a, 2)_{1}}\left(j_{a}\right)=\alpha_{r(a, 2)_{2}}\left(j_{a}\right) \quad \text { and } \\
& \alpha_{r(a, 2)_{1}}(n) \neq \alpha_{r(a, 2)_{2}}(n) .
\end{aligned}
$$

We will use $r(a, k)_{1}, r(a, k)_{2}$, for $k \in\{1,2\}$, to enumerate into $A_{n, s+1}$ while maintaining $Q_{e}^{a}$ 's inequality on $y$. For each $x \in \omega$ and $(a, k) \in[h+1, m] \times\{1,2\}$, and each $y \in W^{(a, k)}(x)$, we set $f(y, 0)=r(a, k)_{1}$. Now if we decide that coding should cooperate with $Q_{e}^{a}$ at $s+1=2 t+1$, then we choose $y_{1} \in W^{(a, 1)}\left(k_{t}\right)$ and $y_{2} \in W^{(a, 2)}\left(k_{t}\right)$ such that $f\left(y_{1}, s\right)=f\left(y_{1}, 0\right)$, and $f\left(y_{2}, s\right)=f\left(y_{2}, 0\right)$ and for all $\left(a^{\prime}, k^{\prime}\right) \in[h+1, m] \times\{1,2\}, \quad f\left(g\left(y_{1}, a^{\prime}, k^{\prime}\right), s\right)=f\left(g\left(y_{1}, a^{\prime}, k^{\prime}\right), 0\right)$ and $f\left(g\left(y_{2}, a^{\prime}, k^{\prime}\right), s\right)=f\left(g\left(y_{2}, a^{\prime}, k^{\prime}\right), 0\right)$. We have three possible actions available now;

1. We set $f\left(y_{1}, s+1\right)=r(a, 1)_{2}$ and $f\left(y_{2}, s+1\right)=f\left(y_{2}, s\right)$,

2. We set $f\left(y_{1}, s+1\right)=f(y, s)$ and $f\left(y_{2}, s+1\right)=r(a, 2)_{2}$,

3. We set $f\left(y_{1}, s+1\right)=r(a, 1)_{2}$ and $f\left(y_{2}, s+1\right)=r(a, 2)_{2}$.

The proof of Lemma 1 shows that one of these three courses of action must maintain the inequality on $y$.

Actions taken by $Q_{e}^{a}$ also interact with the coding. $Q_{e}^{a}$ 's action at $s+1$ consists of looking ahead at numbers which may later get enumerated into $A_{i_{a}} \cup A_{j_{a}}$ and possibly putting some of these numbers into $A_{i_{a}, s+1} \cup A_{j_{a}, s+1}$. For each $x$, we must make sure that $Q_{e}^{a}$ 's action does not use up all the elements of $W(x)$ before the stage $s=2 t+1$ such that $x=k_{t}$ (if such a stage exists). To ensure this we place restrictions on the numbers that $Q_{e}^{a}$ is allowed to enumerate. We say that " $Q_{e}^{a}$ has access to $x$ " if we are allowed to define $f(y, s+1) \neq f(y, s)$ for $y \in W(x) \cup$ $C W(x)$ for the sake of $Q_{e}^{a}$. If $Q_{e}^{a}=T_{i}$ and $x \leqslant i$, then we do not allow $Q_{e}^{a}$ to have access to $x$. For $b \in[1, h] \cup([h+1, m] \times\{1,2\})$ we define

$$
\begin{aligned}
G(x, b, i, s+1)=\{ & y \in W^{b}(x): T_{i} \text { caused } f(y, s+1) \neq f(y, s) \text { or } \\
& \exists\left(a^{\prime}, k^{\prime}\right) \in[h+1, m] \times\{1,2\} \\
& \left.\left(T_{i} \text { caused } f\left(g\left(y, a^{\prime}, k^{\prime}\right), s+1\right) \neq f\left(g\left(y, a^{\prime}, k^{\prime}\right), s\right)\right)\right\} .
\end{aligned}
$$

Then if $x>i$ and $b \in[1, h] \cup([h+1, m] \times\{1,2\})$, we restrict $T_{i}$ 's action so that $\left|\bigcup_{s \in \omega} G(x, b, i, s)\right| \leqslant 2^{x-i}$. This is done via the following rules. At stage $s+1=2 t$, if $x \in K_{t}$ then we allow no requirement $Q_{e}^{a}$ access to $x$, and in this way ensure that $G(x, b, i, s)=\varnothing$ for all $b, i$. If $x \notin K_{t}$ and $G\left(x, b, j, s^{\prime}\right)=\varnothing$ for all $j \leqslant i, s^{\prime} \leqslant s$, and $b \in[1, h] \cup([h+1, m] \times\{1,2\})$, then we allow $T_{i}$ to have access to $x$ (provided $i<x)$ and so we allow $T_{i}$ to change $f$ at $s+1$ on a subset of $W(x) \cup C W(x)$, so long as

$$
|G(x, b, i, s+1)| \leqslant 2^{x-i}, \quad \text { for all } b \in[1, h] \cup([h+1, m] \times\{1,2\}) .
$$

We use the process of "freezing $x$ for $T_{i}$ " to ensure that $\left|\bigcup_{s \in \omega} \bigcup_{i \in \omega} G(x, b, i, s)\right| \leqslant$ $2^{x+1}-2$. For $i, s \in \omega$, if

$$
\exists j \leqslant i \exists s^{\prime} \leqslant s \exists b \in[1, h] \cup([h+1, m] \times\{1,2\})\left(G\left(x, b, j, s^{\prime}\right) \neq \varnothing\right)
$$


then we say that " $x$ is frozen for $T_{i}$ at $t$ ", for all $t \geqslant s$. This means that at all $t \geqslant s$, $T_{i}$ does not have access to $x$, and so $G(x, b, i, t)$ will be empty. In this way we will ensure that the maximum number of elements of $W^{b}(x)$, for any $x \in \omega, b \in[1, h]$ $\cup([h+1, m] \times\{1,2\})$, used up by requirements other than coding is

$$
\begin{aligned}
\left|\bigcup_{s \in \omega} \bigcup_{i \in \omega} G(x, b, i, s)\right| & \leqslant \sum_{i \in \omega}\left(\left|\bigcup_{s \in \omega} G(x, b, i, s)\right|\right) \\
& \leqslant \sum_{i<x} 2^{x-i}=2^{x+1}-2 .
\end{aligned}
$$

Since $W^{b}(x)$ contains $2^{x+1}$ elements, there will always be enough for coding. The restrictions placed on $Q_{e}^{a}$ 's action will be expressed more precisely in the definition of a legal move for $Q_{e}^{a}$.

THEOREM 1. If $\mathscr{P}=\left\langle P, \leqslant, \vee, \wedge, 0_{\mathscr{P}}, 1_{\mathscr{P}}\right\rangle$ is a lattice with more than one element, and $\mathscr{P}$ has a finite representation, then $P$ can be embedded into the r.e. tt-degrees by a map which preserves zero and one.

Proof. Let $P=\left\{p_{0}, \ldots, p_{n}\right\}$, with $p_{0}=0_{\mathscr{P}}$ and $p_{n}=1_{\mathscr{P}}$. Let $\Phi=\left\{\alpha_{r}: r \in J\right\}$ be a finite representation for $\mathscr{P}$, with $J=[0, d], \forall r \leqslant d\left(\alpha_{r}:[0, n] \rightarrow[0, d]\right)$, $\forall r \leqslant d\left(\alpha_{r}(0)=0\right)$ and $\forall r_{1}, r_{2} \leqslant d\left(r_{1} \neq r_{2} \rightarrow \alpha_{r_{1}}(n) \neq \alpha_{r_{2}}(n)\right)$. Let

$$
\begin{aligned}
\{D(\langle e, a\rangle) & : e \in \omega, a \in[1, h]\} \\
\cup & \left\{W^{a}(x): x \in \omega, a \in[1, h] \cup([h+1, m] \times\{1,2\})\right\} \\
\cup & \{C W(x): x \in \omega\}
\end{aligned}
$$

be a recursive partition of $\omega$ satisfying (15), (16) and $\left|W^{a}(x)\right|=2^{x+1}$ for $x \in \omega$, $a \in[1, h] \cup([h+1, m] \times\{1,2\})$. Let $g$ be a $1-1$ recursive map such that for each $x \in \omega, g: W(x) \times[h+1, m] \times\{1,2\} \rightarrow C W(x)$, and let $\left\{T_{i}: i \in \omega\right\}$ be an effective listing of the requirements $P_{e}^{a}$ of (13) and $Q_{e}^{a}$ of (14). For $a \in[1, h]$ let $r(a)_{1}$, $r(a)_{2}$ be recursive maps from $[1, h]$ to $[0, d]$ satisfying (19). For $(a, k) \in[h+1, m]$ $\times\{1,2\}$ let $r(a, k)_{1}, r(a, k)_{2}$ be recursive maps from $[h+1, m] \times\{1,2\}$ to $[0, d]$ satisfying (20). Our construction will define a recursive $f: \omega \times \omega \rightarrow[0, d]$. Let $A_{i, s}=\left\{\left\langle x, \alpha_{f\left(x, s^{\prime}\right)}(i)\right\rangle: s^{\prime} \leqslant s\right\}$, for $i \in[0, n]$, and $A_{i}=\bigcup_{s \in \omega} A_{i, s}$.

We define restraint functions $p(i, s), q(i, s)$, and $R(i, s)$ by

$$
\begin{gathered}
p(i, s)=\left\{\begin{array}{cl}
\left|[e]\left(\left\langle y, \alpha_{r(a)_{2}}\left(i_{a}\right)\right\rangle\right)\right| & \text { if } T_{i}=P_{e}^{a} \text { and } \exists y \in D(\langle e, a\rangle) \\
\uparrow & f(y, s)=r(a)_{2} \text { and } \\
& {[e]^{A_{j_{a}, s}}\left(\left\langle y, \alpha_{r(a)_{2}}\left(i_{a}\right)\right\rangle\right) \downarrow=0,} \\
\text { ow, }
\end{array}\right. \\
q(i, s)= \begin{cases}|[e](y)| & \text { if } T_{i}=Q_{e}^{a} \text { and } \\
\{e\}_{s}\left(y^{\prime}\right) \downarrow \text { for all } y^{\prime} \leqslant y \text { and } & {[e]^{A_{i_{a}, s}} \uparrow y=[e]^{A_{j_{a}, s}} \uparrow y \text { and }} \\
& {[e]^{A_{i_{a}, s}}(y) \neq[e]^{A_{j_{a}, s}}(y),} \\
\text { ow, }\end{cases}
\end{gathered}
$$


$R(i, s)=\max (\{p(j, s): p(j, s) \downarrow$ and $j \leqslant i\} \cup\{q(j, s): q(j, s) \downarrow$ and $j \leqslant i\})$. If $\sigma \in d^{<\omega}$, then $A_{i, s+1}(\sigma)$ is the set that would be $A_{i, s+1}$ if we set $f(x, s+1)=$ $\sigma(x)$ for $x<|\sigma|$ and $f(x, s+1)=f(x, s)$ for $x \geqslant|\sigma|$.

We say that " $\sigma$ is a legal move for $T_{i}$ at $s+1=2 t$ " if (21)-(24) below hold.

$\sigma \in d^{<\omega}$.

(22) For all $x<|\sigma|(\sigma(x) \neq f(x, s) \Rightarrow x>R(i, s)$ and $f(x, s)=f(x, 0))$.

If $T_{i}=P_{e}^{a}$, then there is at most one $x$ such that $\sigma(x) \neq$ $f(x, s)$, and for this $x, x \in D(\langle e, a\rangle)$ and $\sigma(x)=r(a)_{2}$. (This clause says that if $P_{e}^{a}$ acts, then it only changes $f$ on one number, and this number is from the appropriate part of the partition of $\omega$.)

(24.1) $(\forall x<|\sigma|)\left(\forall e^{\prime} \in \omega\right)\left(\forall a^{\prime} \in[1, h]\right)\left(\sigma(x) \neq f(x, s)\right.$ and $x \in D\left(\left\langle e^{\prime}, a^{\prime}\right\rangle\right) \Rightarrow$ $P_{e^{\prime}}^{a^{\prime}}=T_{j}$ and $j>i$ ). (This clause says that $Q_{e}^{a}$ can only touch the witnesses attached to positive requirements of lower priority than itself.)

(24.2) Let $B(x, b, \sigma, s)=\left\{y \in W^{b}(x): \sigma(y) \neq f(y, s)\right.$ or $\exists\left(a^{\prime}, k^{\prime}\right) \in[h+1, m]$ $\left.\times\{1,2\}\left(\sigma\left(g\left(y, a^{\prime}, k^{\prime}\right)\right) \neq f\left(g\left(y, a^{\prime}, k^{\prime}\right), s\right)\right)\right\}$. Then for all $x \in \omega, b \in([1, h]) \cup$ $([h+1, m] \times\{1,2\})$

$$
\begin{aligned}
& \text { if } x \in K_{t} \text {, then } B(x, b, \sigma, s)=\varnothing, \\
& \text { if } x \leqslant i \text {, then } B(x, b, \sigma, s)=\varnothing, \\
& \text { if } x \text { is frozen for } T_{i} \text { at } s, \text { then } B(x, b, \sigma, s)=\varnothing, \\
& \text { otherwise }|B(x, b, \sigma, s)| \leqslant 2^{x-i} .
\end{aligned}
$$

(This clause ensures that $Q_{e}^{a}$ does not use up too many of the witnesses in the sets $W(x)$ for $x \in \omega$. This will ensure that there will always be enough witnesses available for coding $K(x)$.)

(24.3) For all $x, \quad \exists y \in W(x)\left(\sigma(y) \neq f(y, s) \Rightarrow \exists\left(a^{\prime}, k^{\prime}\right) \in[h+1, m] \times\right.$ $\{1,2\}\left(\sigma\left(g\left(y, a^{\prime}, k^{\prime}\right)\right) \neq f\left(g\left(y, a^{\prime}, k^{\prime}\right), 0\right)\right)$. (This clause ensures that our decoding procedure is valid; if $Q_{e}^{a}$ changes $f$ on an element of $W(x)$, then it must also change $f$ on a partner to that element.)

Next we define the phrase " $T_{i}$ requires attention at $s$ ".

$T_{i}=Q_{e}^{a}$ requires attention at $s+1=2 t+1$ if $q(i, s) \downarrow$ and $k_{t}<q(i, s)$.

$T_{i}=P_{e}^{a}$ requires attention at $s+1=2 t+1$ if $p(i, s) \downarrow$ and $k_{t}<p(i, s)$.

$T_{i}=Q_{e}^{a}$ requires attention at $s+1=2 t$ if $q(i, s) \uparrow$ and $\exists \sigma \in d^{<\omega}, \quad \sigma$ is a legal move for $T_{i}$ at $s+1$ and $\exists y\left(\{e\}_{s+1}\left(y^{\prime}\right) \downarrow\right.$ for all $y^{\prime} \leqslant y$ and $[e]^{A_{i_{a r}}(\sigma)} \uparrow y=[e]^{A_{j_{\alpha} s}(\sigma)} \uparrow$ $y$ and $\left.[e]^{A_{i_{a}, s}(\sigma)}(y) \neq[e]^{A_{j_{a} s}(\sigma)}(y)\right)$.

$T_{i}=P_{e}^{a}$ requires attention at $s+1=2 t$ if $p(i, s) \uparrow$ and there is a $y \in D(\langle e, a\rangle)$ such that $y>R(i, s)$ and $[e]^{A_{j_{a} s} s}\left(\left\langle y, \alpha_{r(a)_{2}}\left(i_{a}\right)\right\rangle\right) \downarrow=0$ and $f(y, s)=f(y, 0)=r(a)_{1}$. (Let $\sigma$ be the corresponding legal move.)

We now give the construction of $f$. 
StAGE $s=0$. For all $e \in \omega, a \in[1, h]$, for all $y \in D(\langle e, a\rangle)$, define $f(y, 0)=$ $r(a)_{1}$. For all $x \in \omega, a \in[1, h]$, for all $y \in W^{a}(x)$, define $f(y, 0)=r(a)_{1}$. For all $x \in \omega,(a, k) \in[h+1, m] \times\{1,2\}$, for all $y \in W^{(a, k)}(x)$, for all $z \in W(x)$, define $f(y, 0)=r(a, k)_{1}$, and $f(g(z, a, k), 0)=r(a, k)_{1}$. Since

$$
\left(\bigcup_{x \in \omega} W(x)\right) \cup\left(\bigcup_{x \in \omega} C W(x)\right) \cup\left(\bigcup_{e \in \omega}\left(\bigcup_{a \in[1, h]} D(\langle e, a\rangle)\right)\right)=\omega
$$

we have defined $f(y, 0)$ for all $y \in \omega$.

StAGE $s+1=2 t+1$. $(t \geqslant 0)$ Let $i$ be the least such that $T_{i}$ requires attention at $2 t+1$. We say that $T_{i}$ is active at $s+1$.

If $T_{i}=P_{e}^{a}$, then let $y$ be the least element, if any, of $W^{a}\left(k_{t}\right)$ such that $f(y, s)=f(y, 0)$ and for all $\left(a^{\prime}, k\right) \in[h+1, m] \times\{1,2\}, f\left(g\left(y, a^{\prime}, k\right), s\right)=$ $f\left(g\left(y, a^{\prime}, k\right), 0\right)$. Define $f(y, s+1)=r(a)_{2}$ and $f\left(y^{\prime}, s+1\right)=f\left(y^{\prime}, s\right)$ for all $y^{\prime} \neq$ $y$. If no such $y$ exists, then define $f(x, s+1)=f(x, s)$ for all $x \in \omega$.

If $T_{i}=Q_{e}^{a}$, then let $y_{1}, y_{2}$ be the least elements, if any, of $W^{(a, 1)}\left(k_{t}\right), W^{(a, 2)}\left(k_{t}\right)$, respectively, such that $f\left(y_{1}, s\right)=f\left(y_{1}, 0\right), f\left(y_{2}, s\right)=f\left(y_{2}, 0\right)$, and for all $\left(a^{\prime}, k\right) \in$ $[h+1, m] \times\{1,2\}, \quad f\left(g\left(y_{1}, a^{\prime}, k\right), s\right)=f\left(g\left(y_{1}, a^{\prime}, k\right), 0\right)$ and $f\left(g\left(y_{2}, a^{\prime}, k\right), s\right)=$ $f\left(g\left(y_{2}, a^{\prime}, k\right), 0\right)$. Let $z$ be such that for all $z^{\prime} \leqslant z\left(\{e\}_{s}\left(z^{\prime}\right) \downarrow\right)$ and $[e]^{A_{i_{a} s}} \backslash z=$ $[e]^{A_{j a s},} \uparrow z$ and $[e]^{A_{i a s} s}(z) \neq[e]^{A_{j a s} s}(z)$.

Let $\sigma_{1}, \sigma_{2}, \sigma_{3} \in d^{<\omega}$ have length $1+\max \left\{y_{1}, y_{2}\right\}$ and be defined by

$$
\begin{array}{r}
\sigma_{1}\left(y_{1}\right)=r(a, 1)_{2}, \sigma_{1}(x)=f(x, s) \text { for all } x \neq y_{1}, x<\left|\sigma_{1}\right|, \\
\sigma_{2}\left(y_{2}\right)=r(a, 2)_{2}, \sigma_{2}(x)=f(x, s) \text { for all } x \neq y_{2}, x<\left|\sigma_{2}\right|, \\
\sigma_{3}\left(y_{1}\right)=r(a, 1)_{2}, \sigma_{3}\left(y_{2}\right)=r(a, 2)_{2}, \sigma_{3}(x)=f(x, s) \\
\text { for all } x \neq y_{1}, y_{2}, x<\left|\sigma_{3}\right| .
\end{array}
$$

Choose $\tau=\sigma_{1}, \sigma_{2}$ or $\sigma_{3}$ so that

$$
[e]^{A_{i_{u}, s+1}(\tau)}(z) \neq[e]^{A_{j_{u} s+1}(\tau)}(z),
$$

and define $f(x, s+1)=\tau(x)$ for all $x<|\tau|, f(x, s+1)=f(x, s)$ for all $x \geqslant|\tau|$. (Lemma 1 will show that one of $\sigma_{1}, \sigma_{2}, \sigma_{3}$ must satisfy (28).)

If there are no such $y_{1}, y_{2}$, then define $f(x, s+1)=f(x, s)$ for all $x \in \omega$. If no $T_{i}$ requires attention at $s+1$, then let $y \in W\left(k_{t}\right)$ be the least such that $y$ is reserved for $a=1$ and $f(y, s)=f(y, 0)$ and for all $\left(a^{\prime}, k\right) \in[h+1, m] \times\{1,2\}$, $f\left(g\left(y, a^{\prime}, k\right), s\right)=f\left(g\left(y, a^{\prime}, k\right), 0\right)$. Define $f(y, s+1)=r(1)_{2}$, and $f(x, s+1)=$ $f(x, s)$ for all $x \neq y$.

STAGE $s+1=2 t .(t \geqslant 1)$ Let $i$ be the least such that $T_{i}$ requires attention at $s+1=2 t$. We say that $T_{i}$ is active at $s+1$. If there is no such $T_{i}$, then go on to stage $s+2$. Otherwise, let $\sigma$ be the associated legal move. Define $f(y, s+1)=\sigma(y)$ for all $y<|\sigma|$, and $f(y, s+1)=f(y, s)$ for all $y \geqslant|\sigma|$. If $y<|\sigma|$ and $\sigma(y) \neq f(y, s)$ and $y \in W(x) \cup C W(x)$, then " $x$ is frozen for $T_{j}$ at $s^{\prime \prime}$, for all $s^{\prime} \geqslant s+1$ and all $j \in[i, x]$.

This ends the construction of $f$. 
LEMMA 1. If $[e]^{A_{i_{\alpha} .}}(z) \downarrow \neq[e]^{A_{j_{a} . s}}(z) \downarrow$ and $\sigma_{1}, \sigma_{2}$ and $\sigma_{3}$ are defined by (25), (26) and (27), then (28) holds for $\tau=$ one of $\sigma_{1}, \sigma_{2}, \sigma_{3}$.

Proof. Suppose (28) fails for $\sigma_{1}$ and $\sigma_{2}$, and that $b=[e]^{A_{i_{a}} \cdot s}(z)$ and $1-b=$ $[e]^{A_{j_{a}} s}(z)$. By definition, $\alpha_{r(a, 1)_{1}}\left(i_{a}\right)=\alpha_{r(a, 1)_{2}}\left(i_{a}\right)$ and $\alpha_{r(a, 2)_{1}}\left(j_{a}\right)=\alpha_{r(a, 2)_{2}}\left(j_{a}\right)$. So, $A_{i_{a}, s}\left(\sigma_{1}\right)=A_{i_{a}, s}$, and $A_{j_{a} s}\left(\sigma_{2}\right)=A_{j_{a}, s}$, and $A_{i_{a}, s}\left(\sigma_{3}\right)=A_{i_{a}, s}\left(\sigma_{2}\right)$, and $A_{j_{a}, s}\left(\sigma_{3}\right)=$ $A_{j_{a}, s}\left(\sigma_{1}\right)$. Since (28) fails for $\sigma_{1}$, we get

$$
\begin{aligned}
b & =[e]^{A_{i_{a}, s}}(z)=[e]^{A_{i_{a}, s}\left(\sigma_{1}\right)}(z)=[e]^{A_{j_{a}, s}\left(\sigma_{1}\right)}(z) \\
& =[e]^{A_{j_{a}, s}\left(\sigma_{3}\right)}(z) .
\end{aligned}
$$

Since (28) fails for $\sigma_{2}$, we get

$$
\begin{aligned}
1-b & =[e]^{A_{j_{a}, s}}(z)=[e]^{A_{j_{a}, s}\left(\sigma_{2}\right)}(z)=[e]^{A_{i_{a}, s}\left(\sigma_{2}\right)}(z) \\
& =[e]^{A_{i_{a}, s}\left(\sigma_{3}\right)}(z) .
\end{aligned}
$$

and therefore $[e]^{A_{i_{\alpha}, s}\left(\sigma_{3}\right)}(z) \neq[e]^{A_{j_{\alpha}, s}\left(\sigma_{3}\right)}(z)$, as desired.

LEMMA 2. (a) $A_{0}$ is recursive.

(b) For all $i, j \leqslant n, p_{i} \leqslant p_{j} \Rightarrow A_{i} \leqslant{ }_{\mathrm{tt}} A_{j}$.

(c) For all $i, j, k \leqslant n, p_{i} \vee p_{j}=p_{k} \Rightarrow A_{k} \leqslant{ }_{\mathrm{tt}} A_{i} \oplus A_{j}$.

Proof. As described in the outline before the construction.

LEMMA 3. For each $i \in \omega, T_{i}$ is active at most finitely often, and $\lim _{s} R(i, s)$ exists.

Proof. By induction on $i$. Let $s_{0}$ be such that $(\forall j<i)\left(\forall s \geqslant s_{0}\right)\left(T_{j}\right.$ is not active at $s$ and $\left.R(i-1, s)=R\left(i-1, s_{0}\right)\right)$. Suppose first that $T_{i}=P_{e}^{a}$. If $\exists s>s_{0}(p(i, s) \downarrow)$, then let $s_{1}$ be the least such. Suppose $s>s_{1}, s=2 t$, and $T_{j}$ is active at $s$. Then $j \geqslant i$, since $s>s_{1} \geqslant s_{0}$. Using induction on $s>s_{1}$, we see that $\forall s>s_{1}(p(i, s) \downarrow)$, and so by definition of $T_{i}$ requires attention, we get that $j>i$. Now the definition of a legal move guarantees that $A_{j_{a}, s-1} \uparrow p(i, s-1)=A_{j_{a}, s} \uparrow p(i, s-1)$. If $s>s_{1}$, $s=2 t+1$, and $T_{j}$ is active at $s$, then $j \geqslant i$ (since $s>s_{1}>s_{0}$ ) and the construction guarantees $A_{j_{a}, s} \uparrow p(i, s-1)=A_{j_{s}, s-1} \uparrow p(i, s-1)$. If $j=i$, then $k_{t}<p(i, s-1)$. By induction on $s$ we see that $\forall s \geqslant s_{1}\left(p(i, s)=p\left(i, s_{1}\right)\right)$. Now if $s_{2} \geqslant s_{1}$ is such that $K_{s_{2}} \uparrow p\left(i, s_{1}\right)+1=K \uparrow p\left(i, s_{1}\right)+1$, then $T_{i}$ will never be active after $s_{2}$.

Now suppose that $T_{i}=Q_{e}^{a}$. If $\exists s \geqslant s_{0}(q(i, s) \downarrow)$ then let $s_{1}$ be the least such. Then as above, $\forall s \geqslant s_{1}\left(q(i, s) \downarrow=q\left(i, s_{1}\right)\right)$. If $s_{2} \geqslant s_{1}$ is such that $K_{s_{2}} \uparrow q\left(i, s_{1}\right)+1=K$ i $q\left(i, s_{1}\right)+1$, then $T_{i}$ will never be active after $s_{2}$.

Since for each $i, \exists s_{0}\left(\forall s \geqslant s_{0} p(i, s) \uparrow\right.$ or $\forall s \geqslant s_{0}\left(p(i, s) \downarrow=p\left(i, s_{0}\right)\right)$ and $\left(\forall s \geqslant s_{0}(q(i, s) \uparrow)\right.$ or $\left.\forall s \geqslant s_{0}\left(q(i, s) \downarrow=q\left(i, s_{0}\right)\right)\right)$, we get that $\lim _{s} R(i, s)$ exists.

Lemma 4. For all $a \in[1, h]$, for all $e \in \omega, P_{e}^{a}$ is satisfied.

Proof. Let $i$ be such that $T_{i}=P_{e}^{a}$. Let $s_{0}$ be such that $\forall s \geqslant s_{0}\left(R(i, s)=R\left(i, s_{0}\right)\right.$ and $\forall j \leqslant i\left(T_{j}\right.$ is not active at $\left.\left.s\right)\right)$. Such a stage exists by Lemma 3. Suppose $P_{e}^{a}$ fails,

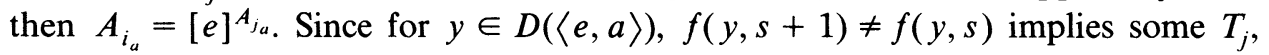
$j \leqslant i$ was active at $s+1$ and by Lemma 3 each $T_{j}$ is active at most finitely often, there are at most finitely many $y \in D(\langle e, a\rangle)$ such that $\exists s \in \omega(f(y, s) \neq f(y, 0))$. 
Let $y$ be the least element of $D(\langle e, a\rangle)$ such that $y>R\left(i, s_{0}\right)$ and $\forall s \in \omega(f(y, s)$ $=f(y, 0))$. Then $\left\langle y, \alpha_{r(a)_{2}}\left(i_{a}\right)\right\rangle \notin A_{i_{a}}$, and since $A_{i_{a}}=[e]^{A_{j a}}$, we get $[e]^{A_{j a}}\left(\left\langle y, \alpha_{r(a)_{2}}\left(i_{a}\right)\right\rangle\right)=0$. If $s_{1}=2 t>s_{0}$ is such that

$$
A_{j_{a}, s_{1}} \uparrow\left|[e]\left(\left\langle y, \alpha_{r(a)_{2}}\left(i_{a}\right)\right\rangle\right)\right|=A_{j_{a}} \uparrow\left|[e]\left(\left\langle y, \alpha_{r(a)_{2}}\right\rangle\right)\right|,
$$

then $T_{i}$ requires attention at $s_{1}$, and since no $T_{j}, j<i$ is active at $s_{1}, T_{i}$ will become active at $s_{1}>s_{0}$. This is a contradiction, so $P_{e}^{a}$ must be satisfied.

Lemma $5 . K \leqslant{ }_{\mathrm{tt}} A_{n}$.

Proof. We show that

$$
\begin{aligned}
& \forall x \in \omega\left(x \in K \leftrightarrow \left(\exists a \in [ 1 , h ] \exists y \in W ^ { a } ( x ) \left(\left\langle y, \alpha_{r(a)_{2}}(n)\right\rangle \in A_{n}\right.\right.\right. \text { and } \\
& \forall\left(a^{\prime}, k^{\prime}\right) \in[h+1, m] \times\{1,2\} \forall r \leqslant d\left(r \neq r\left(a^{\prime}, k^{\prime}\right)_{1} \rightarrow\right. \\
& \left.\left.\left.\left\langle g\left(y, a^{\prime}, k^{\prime}\right), \alpha_{r}(n)\right\rangle \notin A_{n}\right)\right)\right)
\end{aligned}
$$

or

$$
\begin{gathered}
\left(\exists(a, k) \in[h+1, m] \times\{1,2\} \exists y \in W^{(a, k)}(x)\left(\left\langle y, \alpha_{r(a, k)_{2}}(n)\right\rangle \in A_{n}\right. \text { and }\right. \\
\forall\left(a^{\prime}, k^{\prime}\right) \in[h+1, m] \times\{1,2\} \forall r \leqslant d\left(r \neq r\left(a^{\prime}, k^{\prime}\right)_{1} \rightarrow\right. \\
\left.\left.\left.\left.\left\langle g\left(y, a^{\prime}, k^{\prime}\right), \alpha_{r}(n)\right\rangle \notin A_{n}\right)\right)\right)\right) .
\end{gathered}
$$

This provides the desired tt-reduction. We first show that for all $t, W\left(k_{t}\right)$ contains enough elements to carry out the prescribed coding action at stage $2 t+1$ in the construction. We say that $y \in W(x)$ is "free for coding at $s+1$ " if $f(y, s)=f(y, 0)$ and for all $(a, k) \in[h+1, m] \times\{1,2\}, f(g(y, a, k), s)=f(g(y, a, k), 0)$.

ClaIm 5.1. If $x=k_{t}$ and $a \in[1, h]$, then there is a $y \in W^{a}(x)$ such that $y$ is free for coding at $2 t+1$. If $(a, k) \in[h+1, m] \times\{1,2\}$, then there is a $y \in W^{(a, k)}(x)$ such that $y$ is free for coding at $2 t+1$.

PROOF. For $a \in[1, h]$ we define

$$
\begin{array}{r}
V^{a}(x, s)=\left\{y \in W^{a}(x): f(y, s) \neq f(y, 0) \text { or } \exists\left(a^{\prime}, k^{\prime}\right) \in[h+1, m] \times\{1,2\}\right. \\
\left.f\left(g\left(y, a^{\prime}, k^{\prime}\right), s\right) \neq f\left(g\left(y, a^{\prime}, k^{\prime}\right), 0\right)\right\} .
\end{array}
$$

We must show that there is a $y \in W^{a}(x)$ such that $y \notin V^{a}(x, 2 t)$ (where $\left.x=k_{t}\right)$. Since $\left|W^{a}(x)\right|=2^{x+1}$, it suffices to show $\left|V^{a}(x, 2 t)\right| \leqslant 2^{x+1}-2$. Note that $V^{a}(x, 2 t)=\bigcup_{s^{\prime}<2 t}\left(V^{a}\left(x, s^{\prime}+1\right)-V^{a}\left(x, s^{\prime}\right)\right)$. Aiso note that if $s^{\prime}+1=2 t^{\prime}+1 \leqslant$ $2 t$, then $k_{t^{\prime}} \neq x$ (the enumeration is 1-1) and so our construction ensures

$$
V^{a}\left(x, s^{\prime}+1\right)-V^{a}\left(x, s^{\prime}\right)=\varnothing .
$$

If $s^{\prime}+1=2 t^{\prime} \leqslant 2 t$ and $V^{a}\left(x, s^{\prime}+1\right)-V^{a}\left(x, s^{\prime}\right) \neq \varnothing$, then some $T_{i}=Q_{e}^{a^{\prime}}$ was active at $s^{\prime}+1$, and executed a legal move. Since $V^{a}\left(x, s^{\prime}+1\right)-V^{a}\left(x, s^{\prime}\right) \neq \varnothing$, the definition of a legal move guarantees that $i<x$, and $\left|V^{a}\left(x, s^{\prime}+1\right)-V^{a}\left(x, s^{\prime}\right)\right|$ $\leqslant 2^{x-i}$. The process of freezing $x$ for $T_{i}$ guarantees that for each $i$ there is at most 
one $s^{\prime}$ such that $\left(T_{i}\right.$ is active at $s^{\prime}+1$ and $\left.V^{a}\left(x, s^{\prime}+1\right)-V^{a}\left(x, s^{\prime}\right) \neq \varnothing\right)$. Therefore,

$$
\left|V^{a}(x, 2 t)\right| \leqslant \sum_{i<x} 2^{x-i}=2^{x+1}-2
$$

If we replace $a$ everywhere by $(a, k) \in[h+1, m] \times\{1,2\}$, then the above argument shows that there is a $y \in W^{(a, k)}\left(k_{t}\right)$ which is free for coding at $2 t+1$.

The construction guarantees that if $x \in K_{t}$, then for all $s \geqslant 2 t+2$, for all $y \in W(x) \cup C W(x), f(y, s+1)=f(y, s)$. This, together with claim 5.1 establishes " $\rightarrow$ " for (29). We establish " $\leftarrow$ " for (29) by showing

Claim 5.2. If $s+1=2 t$ and $x \notin K_{t}$ and if $\exists y \in W(x)(f(y, s+1) \neq f(y, s))$, then

$$
\exists\left(a^{\prime}, k^{\prime}\right) \in[h+1, m] \times\{1,2\}\left(f\left(g\left(y, a^{\prime}, k^{\prime}\right), s+1\right) \neq f\left(g\left(y, a^{\prime}, k^{\prime}\right), 0\right)\right) .
$$

Proof. If $y \in W(x)$ and $f(y, s+1) \neq f(y, s)$, then some $T_{i}=Q_{e}^{a}$, with $i<x$ must have been active at $s+1$ and executed a legal move. The definition of a legal move, in particular (24.3), guarantees that the claim holds.

Now suppose $x \notin K$. If $\forall a \in[1, h] \quad \forall y \in W^{a}(x)\left(\left\langle y, \alpha_{r(a)_{2}}(n)\right\rangle \notin A_{n}\right)$ and $\forall(a, k) \in[h+1, m] \times\{1,2\} \quad \forall y \in W^{(a, k)}(x)\left(\left\langle y, \alpha_{r(a, k)_{2}}(n)\right\rangle \notin A_{n}\right)$, then (29) is satisfied. Otherwise, if $a \in[1, h]$ and $y \in W^{a}(x)$ is such that $\left\langle y, \alpha_{r(a)_{2}}(n)\right\rangle \in A_{n}$, then since $\alpha_{f(y, 0)}(n) \neq \alpha_{r(a)_{2}}(n)$, there is an $s+1$ such that $f(y, s+1) \neq f(y, s)$. Since $x \notin K, s=2 t$ and $x \notin K_{t}$, and so Claim 5.2 guarantees $\exists\left(a^{\prime}, k^{\prime}\right) \in[h+$ $1, m] \times\{1,2\} \quad \exists r \in J\left(r \neq f\left(g\left(y, a^{\prime}, k^{\prime}\right), 0\right)\right)$ and $\left\langle g\left(y, a^{\prime}, k^{\prime}\right), \alpha_{r}(n)\right\rangle \in A_{n}$. If we replace $a$ everywhere by $(a, k)$ in the above argument, then we have established (29).

LEMMA 6. For all $a \in[h+1, m]$, for all $e \in \omega, Q_{e}^{a}$ is satisfied.

Proof. Suppose that $[e]^{A_{i_{a}}}=[e]^{A_{j_{a}}}=Y$. We want to show $Y \leqslant{ }_{\mathrm{tt}} A_{k_{a}}$. Let $i$ be such that $T_{i}=Q_{e}^{a}$. Let $s_{0}$ be such that $\forall s \geqslant s_{0} \forall j \leqslant i\left(T_{j}\right.$ is not active at $s$ and $\left.R(i, s)=R\left(i, s_{0}\right)=R(i)\right)$. Let

$$
q=1+\max \left(\{i, R(i)\} \cup\left\{x: x \text { is frozen for } T_{i} \text { at } s_{0}\right\}\right)
$$

Let $s_{1} \geqslant s_{0}$ be such that $K_{s_{1}} \uparrow q=K \uparrow q$. In (30) we outline a reduction procedure from an arbitrary oracle, $B$. This reduction procedure will be total for all oracles, $B$, and so by Nerode's result [6, p. 143] we have a tt-reduction. We first outline the reduction procedure for input $y$ and then verify that when $B=A_{k_{a}}$ the procedure computes $Y$.

Find a stage $s_{2}=2 t \geqslant s_{1}$ such that for all $y^{\prime} \leqslant y\left(\{e\}_{s_{2}}\left(y^{\prime}\right) \downarrow\right)$.

See if there is a $\sigma \in d^{<\omega}$ such that

$|\sigma|=|[e](y)|$ and $B \uparrow\langle|[e](y)|, d\rangle=A_{k_{a}, s_{2}}(\sigma) \uparrow\langle|[e](y)|, d\rangle$,

and for all $z<|\sigma|$, if $\left(\forall r \in J\left(\left\langle z, \alpha_{r}\left(k_{a}\right)\right\rangle \in B \Rightarrow \alpha_{r}\left(k_{a}\right)=\right.\right.$ $\alpha_{f(z, 0)}\left(k_{a}\right)$ or $\left.\left.\alpha_{r}\left(k_{a}\right)=\alpha_{f\left(z, s_{2}\right)}\left(k_{a}\right)\right)\right)$ then $\sigma(z)=f\left(z, s_{2}\right)$. If so then output $[e]^{A_{i_{\alpha}, s_{2}}(\sigma)}(y)$, if not then output $y$. 
We now show that $[e]^{A_{i_{u}, s_{2}}(\sigma)}(y)=[e]^{A_{i_{a}}}(y)=Y(y)$, where $\sigma$ is the string used in (30). Let $s_{3} \geqslant s_{2}$ be such that $A_{i_{a}, s_{3}} \uparrow\langle|[e](y)|, d\rangle=A_{i_{a}} \uparrow\langle\|[e](y) \mid, d\rangle$. Define $\tau \in d^{<\omega}$ so that $|\tau|=|[e](y)|$ and $\tau(x)=f\left(x, s_{3}\right)$ for all $x<|\tau|$. Then $[e]^{A_{i_{a}}}(y)=$ $[e]^{A_{i_{a}, s_{2}}(\tau)}(y)$, so it suffices to show

$$
[e]^{A_{i_{a}, s_{2}}(\sigma)}(y)=[e]^{A_{i_{a}, s_{2}}(\tau)}(y) .
$$

Notice that for all $x<|[e](y)|=|\sigma|=|\tau|, \alpha_{\sigma(x)}\left(k_{a}\right)=\alpha_{\tau(x)}\left(k_{a}\right)$. This is true since if $\alpha_{\sigma(x)}\left(k_{a}\right) \neq \alpha_{f\left(x, s_{2}\right)}\left(k_{a}\right)$ then the definition of $\sigma$ in (30) guarantees that $f\left(x, s_{2}\right)=$ $f(x, 0)$ and $\exists s>s_{2}\left(f(x, s) \neq f\left(x, s_{2}\right)\right.$, and $\left.\alpha_{f(x, s)}\left(k_{a}\right) \neq \alpha_{f(x, 0)}\left(k_{a}\right)\right)$. But since $p_{k_{a}}$ $\leqslant p_{i_{a}}$, (1) implies that $\alpha_{f(x, s)}\left(i_{a}\right) \neq \alpha_{f(x, 0)}\left(i_{a}\right)$, and so $s \leqslant s_{3}$ and so $f(x, s)=$ $f\left(x, s_{3}\right)=\tau(x)$. The definition of $\sigma$ also guarantees that if $\alpha_{\sigma(x)}\left(k_{a}\right) \neq \alpha_{f\left(x, s_{2}\right)}\left(k_{a}\right)$, then $\alpha_{\tau(x)}\left(k_{a}\right)=\alpha_{f\left(x, s_{2}\right)}\left(k_{a}\right)=\alpha_{\sigma(x)}\left(k_{a}\right)$.

Now since $\alpha_{\sigma(x)}\left(k_{a}\right)=\alpha_{\tau(x)}\left(k_{a}\right)$, (3) implies that for each $x<|\sigma|$ such that $\sigma(x) \neq \tau(x)$ we can find a sequence $r_{x_{0}}, \ldots, r_{x_{m_{x}}}$ from $J$ such that $r_{x_{0}}=\sigma(x)$, $r_{x_{m_{x}}}=\tau(x)$, and for $b<m_{x}-1, b$ even,

$$
\alpha_{r_{x_{b}}}\left(i_{a}\right)=\alpha_{r_{x_{b+1}}}\left(i_{a}\right) \text { and } \alpha_{r_{x_{b+1}}}\left(j_{a}\right)=\alpha_{r_{x_{b+2}}}\left(j_{a}\right) \text {. }
$$

Let $p=\max \left\{m_{x}: \sigma(x) \neq \tau(x)\right\}$ and define $\sigma_{0}, \ldots, \sigma_{p} \in d^{<\omega}$ of length $|\sigma|$ as follows for $b \in[0, p]$

$$
\sigma_{b}(x)= \begin{cases}r_{x_{b}} & \text { if } b \leqslant m_{x} \text { and } \sigma(x) \neq \tau(x), \\ r_{x_{m_{x}}} & \text { if } b \geqslant m_{x} \text { and } \sigma(x) \neq \tau(x), \\ \sigma(x) & \text { if } \sigma(x)=\tau(x) .\end{cases}
$$

Now we have $\sigma_{0}=\sigma, \sigma_{p}=\tau$, and for $b \in[0, p-1], b$ even,

$$
A_{i_{a}, s_{2}}\left(\sigma_{b}\right) \uparrow\langle|[e](y)|, d\rangle=A_{i_{a}, s_{2}}\left(\sigma_{b+1}\right) \uparrow\langle|[e](y)|, d\rangle
$$

and

$$
A_{j_{a}, s_{2}}\left(\sigma_{b+1}\right) \uparrow\langle|[e](y)|, d\rangle=A_{j_{a}, s_{2}}\left(\sigma_{b+2}\right) \uparrow\langle|[e](y)|, d\rangle .
$$

If we can show, for $b \in[0, p]$

$$
[e]^{A_{i_{a} \cdot s_{2}}\left(\sigma_{b}\right)}(y)=[e]^{A_{j_{a} \cdot s_{2}}\left(\sigma_{b}\right)}(y),
$$

then by combining (32), (33) and (34) we get

$$
\begin{aligned}
{[e]^{A_{i_{a}, s_{2}}(\sigma)}(y) } & =[e]^{A_{i_{a}, s_{2}}\left(\sigma_{0}\right)}(y)=[e]^{A_{i_{\alpha}, s_{2}}\left(\sigma_{1}\right)}(y) \\
& =[e]^{A_{j_{\alpha}, s_{2}}\left(\sigma_{1}\right)}(y)=[e]^{A_{j_{a}, s_{2}}\left(\sigma_{2}\right)}(y)=[e]^{A_{i_{a}, s_{2}}\left(\sigma_{2}\right)}(y) \\
& =\cdots=[e]^{A_{j_{u}, s_{2}}\left(\sigma_{p}\right)}(y)=[e]^{A_{i_{a}, s_{2}}\left(\sigma_{p}\right)}(y) \\
& =[e]^{A_{i_{\alpha}, s_{2}}(\tau)}(y) .
\end{aligned}
$$

The outside terms in this string of equalities give (31), and hence the lemma.

Suppose that (34) fails for some $b \in[0, p]$, and let $b$ be the least such. We will construct a legal move for $T_{i}$ at $s_{2}, \mu$, from $\sigma_{b}$, such that $[e]^{A_{i_{\alpha} \cdot s_{2}}(\mu)}(y) \neq[e]^{A_{j_{\alpha}, s_{2}}(\mu)}(y)$, and hence $T_{i}$ will require attention and become active at $s_{2}$, a contradiction. 
We obtain $\mu$ by patching up $\sigma_{b}$ to satisfy (24.3). Let $\left\{\left\langle y_{1}, x_{1}\right\rangle, \ldots,\left\langle y_{u}, x_{u}\right\rangle\right\}$ be a list of all the pairs $\left\langle y_{i}, x_{i}\right\rangle$ such that $y_{i} \in W\left(x_{i}\right)$ and $\sigma_{b}\left(y_{i}\right) \neq f\left(y_{i}, s_{2}\right)$ and for all $\left(a^{\prime}, k^{\prime}\right) \in[h+1, m] \times\{1,2\}, \sigma_{b}\left(g\left(y_{i}, a^{\prime}, k^{\prime}\right)\right)=f\left(g\left(y_{i}, a^{\prime}, k^{\prime}\right), 0\right)=r\left(a^{\prime}, k^{\prime}\right)_{1}$. For each $i \leqslant u+1$ we will define a $\sigma_{b}^{i}$ so that $\sigma_{b}^{0}=\sigma_{b}$ and for $i \leqslant u$

$$
\left\{z: \sigma_{b}^{i+1}(z) \neq \sigma_{b}^{i}(z)\right\} \subseteq\left\{g\left(y_{i}, a, k\right): k \in\{1,2\}\right\}
$$

and

$$
[e]^{A_{i_{a}, s_{2}}\left(\sigma_{b}^{i+1}\right)}(y) \neq[e]^{A_{j_{u}, s_{2}}\left(\sigma_{b}^{i+1}\right)}(y) .
$$

Suppose we have defined $\sigma_{b}^{i}$ satisfying (35) and (36). To get $\sigma_{b}^{i+1}$, let $\tau_{1}, \tau_{2}$, $\tau_{3} \in d^{<\omega}$ have length $\left|\sigma_{b}\right|$ and be defined by

$$
\begin{aligned}
& \tau_{1}(x)= \begin{cases}r(a, 1)_{2} & \text { if } x=g\left(y_{i}, a, 1\right), \\
\sigma_{b}^{i}(x) & \text { ow }\end{cases} \\
& \tau_{2}(x)= \begin{cases}r(a, 2)_{2} & \text { if } x=g\left(y_{i}, a, 2\right), \\
\sigma_{b}^{i}(x) & \text { ow, }\end{cases} \\
& \tau_{3}(x)= \begin{cases}r(a, 1)_{2} & \text { if } x=g\left(y_{i}, a, 1\right), \\
r(a, 2)_{2} & \text { if } x=g\left(y_{i}, a, 2\right), \\
\sigma_{b}^{i}(x) & \text { ow, }\end{cases}
\end{aligned}
$$

then (35) holds for $\sigma_{b}^{i+1}=$ any of $\tau_{1}, \tau_{2}$ or $\tau_{3}$, and just as in the proof of Lemma 1, (36) must hold for $\sigma_{b}^{i+1}=$ one of $\tau_{1}, \tau_{2}$ or $\tau_{3}$. So we choose $\sigma_{b}^{i+1}=\tau_{1}, \tau_{2}$ or $\tau_{3}$ to make (36) hold.

Let $\mu=\sigma_{b}^{u+1}$. We show that $\mu$ is a legal move for $T_{i}$ at $s_{2}$ by verifying (22)-(24) for $\mu, T_{i}$ and $s_{2}$.

Claim 6.1. For all $y<|\mu|$, if $\mu(y)=\sigma_{b}(y)$ and $\mu(y) \neq f\left(y, s_{2}\right)$, then there is an $s \geqslant s_{2}$ such that $f(y, s+1) \neq f(y, s)$.

Proof. Suppose $\mu(y)=\sigma_{b}(y)$ and $\mu(y) \neq f\left(y, s_{2}\right)$. If $\sigma(y)=\tau(y)$, then $\sigma_{b}(y)$ $=\tau(y)=f\left(y, s_{3}\right)$, and $f\left(y, s_{3}\right) \neq f\left(y, s_{2}\right)$, so for some $s \geqslant s_{2} f(y, s+1) \neq f(y, s)$. As noted above, $\forall x<|\sigma|, \alpha_{\sigma(x)}\left(k_{a}\right)=\alpha_{\tau(x)}\left(k_{a}\right)$, so if $\sigma(y) \neq \tau(y)$, then $\sigma(y)=$ $f\left(y, s_{2}\right)$. Thus we have $f\left(y, s_{2}\right) \neq f\left(y, s_{3}\right)$, so for some $s \geqslant s_{2} f(y, s+1) \neq f(y, s)$.

VERIFICATION OF (22)-(24).

(22) We need to show that if $\mu(y) \neq f\left(y, s_{2}\right)$, then $y>R\left(i, s_{2}\right)$ and $f\left(y, s_{2}\right)=$ $f(y, 0)$. Suppose $\mu(y) \neq f\left(y, s_{2}\right)$. If $\mu(y)=\sigma_{b}(y)$, then Claim 6.1 guarantees that $\exists s \geqslant s_{2}, f(y, s+1) \neq f(y, s)$. If $s+1=2 t$, then some $T_{j}$ is active at $s+1$, and since $s+1 \geqslant s_{2}>s_{0}, j$ must be greater than $i$. $T_{j}$ must have executed a legal move at $s+1$, so (22) for $T_{j}$ at $s+1$ implies that $y>R(j, s+1) \geqslant R(i, s+1)=$ $R\left(i, s_{2}\right)$. Also, if $f(y, s+1) \neq f(y, s)$ then (22) for $T_{j}$ at $s+1$ implies that $f(y, s)=f\left(y, s_{2}\right)=f(y, 0)$. If $s+1=2 t+1$, then since $K_{s_{1}} \uparrow R(i)=K \uparrow R(i)$, $k_{t}>R(i)$. Since $y \in W\left(k_{t}\right)$ and all elements of $W\left(k_{t}\right)$ are greater than $k_{t}$, we get $y>R(i)=R\left(i, s_{2}\right)$, and again $f(y, s)=f\left(y, s_{2}\right)=f(y, 0)$. Thus we get (22) for $\mu(y)$ if $\mu(y)=\sigma_{b}(y)$. If $\mu(y) \neq \sigma_{b}(y)$, then $y=g(z, a, k)$ for some $k \in\{1,2\}$ and $z$ such that $\sigma_{b}(z) \neq f\left(z, s_{2}\right)$. Since $\mu(z)=\sigma_{b}(z)$, the above argument shows that $z>R(i)$. Since $g(z, a, k)>z$, we get $y>z>R(i)=R\left(i, s_{2}\right)$. Our choice of the $\sigma_{b}^{i}$ for $i \leqslant u+1$ guarantees that $f\left(y, s_{2}\right)=f(y, 0)$, so we get (22) for $\mu(y) \neq \sigma_{b}(y)$. 
(23) This condition applies only when $T_{i}=P_{e^{\prime}}^{a^{\prime}}$, and so is satisfied vacuously, since $T_{i}=Q_{e}^{a}$.

(24.1) We need to show that if $\mu(y) \neq f\left(y, s_{2}\right)$ and $y \in D\left(\left\langle e^{\prime}, a^{\prime}\right\rangle\right)$, then $P_{e^{\prime}}^{a^{\prime}}=T_{j}$ with $j>i$. If $\mu(y) \neq f\left(y, s_{2}\right)$ and $y \in D\left(\left\langle e^{\prime}, a^{\prime}\right\rangle\right)$, then $\mu(y)=\sigma_{b}(y)$, and by Claim 6.1, $\exists s \geqslant s_{2}(f(y, s+1) \neq f(y, s))$. Since $y \in D\left(\left\langle e^{\prime}, a^{\prime}\right\rangle\right)$, it must be the case that $s+1=2 t$ and some $T_{j}$ is active at $s+1$. Since $s+1>s_{0}, j>i$. So if $T_{j}=P_{e^{\prime}}^{a^{\prime}}$, then (24.1) holds for $\mu(y)$ and $T_{i}$ at $s_{2}$. Otherwise, $T_{j}=Q_{e^{\prime \prime}}^{a^{\prime \prime}}$ for some $e^{\prime \prime}$, $a^{\prime \prime}$, and $P_{e^{\prime}}^{a^{\prime}}=T_{k}$. Then since $T_{j}$ must have made a legal move at $s+1,(24.1)$ for $T_{j}$ at $s+1$ implies $k>j>1$, so $k>i$ and (24.1) holds for $\mu(y)$ and $T_{i}$, at $s_{2}$.

(24.2) We need to show that the sets $B\left(x, c, \mu, s_{2}\right)=\left\{y \in W^{c}(x): \mu(y) \neq f\left(y, s_{2}\right)\right.$ or $\left.\exists\left(a^{\prime}, k^{\prime}\right) \in[h+1, m] \times\{1,2\}\left(\mu\left(g\left(y, a^{\prime}, k^{\prime}\right)\right) \neq f\left(g\left(y, a^{\prime}, k^{\prime}\right), s_{2}\right)\right)\right\}$ have the correct cardinalities. Fix $x \in \omega$ and $c \in[1, h] \cup[h+1, m] \times\{1,2\}$ and consider $B\left(x, c, \mu, s_{2}\right)$. If $y \in B\left(x, c, \mu, s_{2}\right) \neq \varnothing$, then either $\mu(y)=\sigma_{b}(y) \neq f\left(y, s_{2}\right)$ or $\left(\mu(y)=\sigma_{b}(y)=f\left(y, s_{2}\right)\right.$ and $\mu\left(g\left(y, a^{\prime}, k^{\prime}\right)\right) \neq f\left(g\left(y, a^{\prime}, k^{\prime}\right), s_{2}\right)$ for some $\left(a^{\prime}, k^{\prime}\right)$ $\in[h+1, m] \times\{1,2\})$. If $\mu(y)=\sigma_{b}(y)=f\left(y, s_{2}\right)$, then our definition of $\mu$ guarantees that $\mu\left(g\left(y, a^{\prime}, k^{\prime}\right)\right)=\sigma_{b}\left(g\left(y, a^{\prime}, k^{\prime}\right)\right)$, and so we get that $\sigma_{b}\left(g\left(y, a^{\prime}, k^{\prime}\right)\right)$ $\neq f\left(g\left(y, a^{\prime}, k^{\prime}\right), s_{2}\right)$. Thus by Claim 6.1 we know that if $y \in B\left(x, c, \mu, s_{2}\right)$ then there must be an $s \geqslant s_{2}$ such that either $f(y, s+1) \neq f(y, s)$ or $f\left(g\left(y, a^{\prime} k^{\prime}\right), s+1\right)$ $\neq f\left(g\left(y, a^{\prime}, k^{\prime}\right), s\right)$. So one of (37) or (38) below must hold.

$$
\exists s>s_{2}\left(s=2 t+1 \text { and } x=k_{t}\right) \text {, or }
$$

$\exists s>s_{2} \exists j<x \exists \eta \in d^{<\omega}(s=2 t$ and $\eta$ is a legal move

for $T_{j}$ at $s$ and $\left.y \in B(x, c, \eta, s)\right)$.

Condition (37) can occur at most once, and can contribute at most one element to $B\left(x, c, \mu, s_{2}\right)$. Condition (38) can occur at most once for each $j<x$, and so for a fixed $j<x$ can contribute at most $2^{x-j}$ elements to $B\left(x, c, \mu, s_{2}\right)$. Since $s_{2} \geqslant s_{0}$, no $T_{k}$ with $k \leqslant i$ can be active at $s \geqslant s_{2}$, so we need only consider $T_{j}$ such that $i<j<x$, and we get

$$
\left|B\left(x, c, \mu, s_{2}\right)\right| \leqslant 1+\sum_{i<j<x} 2^{x-j}=1+\left(2^{x-i}-2\right) \leqslant 2^{x-i} .
$$

Also notice that if $s_{2}=2 t$ and $x \in K_{t}$, then for all $y \in W(x) \cup C W(x)$, for all $s \geqslant s_{2}, f(y, s)=f\left(y, s_{2}\right)$, and so $\mu(y)=\sigma_{b}(y)=f\left(y, s_{2}\right)$, and so $B\left(x, c, \mu, s_{2}\right)=$ $\varnothing$. If $x \leqslant i$, then since $K_{s_{2}} \uparrow i=K \uparrow i$, and no $T_{j}, j \leqslant i$ is active at $s \geqslant s_{2}$, again we get that $\forall y \in W(x) \cup C W(x) \forall s \geqslant s_{2}\left(f(y, s)=f\left(y, s_{2}\right)\right)$ and so $B\left(x, c, \mu, s_{2}\right)=$ $\varnothing$. If $x$ is frozen for $T_{i}$ at $s_{2}$, let $s^{\prime} \leqslant s_{2}$ be the least such that $x$ first became frozen for $T_{i}$ at $s^{\prime}$. Then some $T_{j}, j \leqslant i$ was active at $s^{\prime}$, and so $s^{\prime}<s_{0}$. But then $x<q$, and so $K_{s_{2}} \uparrow x+1=K \uparrow x+1$. Since $x$ is frozen for $T_{i}$ at $s_{2}, x$ is frozen for $T_{k}$ at $s$, for all $s \geqslant s_{2}$, for all $k(i \leqslant k<x)$. Therefore for all $y \in W(x) \cup C W(x)$, for all $s \geqslant s_{2}, f(y, s+1)=f(y, s)$, and so $B\left(x, c, \mu, s_{2}\right)=\varnothing$. Thus we have shown that (24.2) holds for $\mu$ and $T_{i}$ at $s_{0}$.

(24.3) We need to show for any $x$ that if there is a $y \in W(x)$ such that $\mu(y) \neq f\left(y, s_{2}\right)$, then there is a pair $\left(a^{\prime}, k^{\prime}\right) \in[h+1, m] \times\{1,2\}$ such that $\mu\left(g\left(y, a^{\prime}, k^{\prime}\right)\right) \neq f\left(g\left(y, a^{\prime}, k^{\prime}\right)\right), s_{2}$. Our definition of $\mu$ as a modification of $\sigma_{b}$ ensures that (24.3) holds. 
Thus we have shown that $\mu$ is a legal move for $T_{i}$ at $s_{2}$, and that $[e]^{A_{i_{a}, s_{2}}(\mu)}(y) \neq$ $[e]^{A_{j_{a}, s_{2}}(\mu)}(y)$, so $T_{i}$ becomes active at $s_{2}$, a contradiction. We conclude that $Q_{e}^{a}$ is satisfied.

COROLlaRY 1. Every finite lattice with more than one element can be embedded into the r.e. $t$-degrees preserving least and greatest element.

Proof. The corollary follows from Theorem 1 since every finite lattice has a finite representation [5].

2. Recursively presentable lattices. In this section we will modify the proof of Theorem 1 to show that every recursively presentable lattice can be embedded in the r.e. tt-degrees preserving least and greatest elements. Note that by Shore [7] not every lattice can be embedded in this way; some restriction on the degree of the lattice presentation is necessary.

THEOREM 2. Every lattice, $\mathscr{P}$, which has a recursive presentation and distinct least and greatest elements can be embedded into the r.e. $t$ t-degrees preserving least and greatest elements.

ProOF. We fix some notation first. Let $\mathscr{P}=\left\langle P, \leqslant, \vee, \wedge, 0_{\mathscr{P}}, 1_{\mathscr{P}}\right\rangle$ be a lattice with $0_{\mathscr{P}} \neq 1_{\mathscr{P}}$. Let $\Phi=\left\{\alpha_{r}: r \in \omega\right\}$ be a recursive representation of $\mathscr{P}$. Since $\mathscr{P}$ has a recursive presentation, we know that such a representation exists. Let $P=$ $\left\{p_{i}: i \in \omega\right\}$, with $p_{0}=0_{\mathscr{P}}$, and $p_{1}=1_{\mathscr{P}}$. We assume, w.1.o.g., that $\forall r \in \omega, \alpha_{r}(0)=0$ and $\forall r_{1}, r_{2} \in \omega\left(r_{1} \neq r_{2} \Rightarrow \alpha_{r_{1}}(1) \neq \alpha_{r_{2}}(1)\right)$. Let $\left\{R_{a}: a \in \omega\right\}$ be an effective listing of all the relations " $p_{i} \nless p_{j}$ " or " $p_{i} \wedge p_{j}=p_{k}$ " in $P$, i.e. $R_{a}$ is " $p_{i_{a}} \nless p_{j_{a}}$ " or " $p_{i_{a}} \wedge p_{j_{a}}=p_{k_{a}}$ ", and if $p_{i} \nless p_{j}$ then $\exists a \in \omega, R_{a}=" p_{i} \nless p_{j}$ ", and if $p_{i} \mid p_{j}$ and $p_{i} \wedge p_{j}=p_{k}$, then $\exists a \in \omega, R_{a}=" p_{i} \wedge p_{j}=p_{k}$ ". Define

$$
\begin{gathered}
H_{x}=\left\{a<x: R_{a}=" p_{i_{a}} \nless p_{j_{a}} "\right\}, \quad h_{x}=\left|H_{x}\right|, \\
M_{x}=\left\{a<x: R_{a}={ }^{\prime} p_{i_{a}} \wedge p_{j_{a}}=p_{k_{a}} "\right\}, \quad m_{x}=\left|M_{x}\right| .
\end{gathered}
$$

For $a \in \cup_{x \in \omega} H_{x}$, define $P_{e}^{a}$ as in (13), and for $a \in \bigcup_{x \in \omega} M_{x}$, define $Q_{e}^{a}$ as in (14). Let $\left\{T_{i}: i \in \omega\right\}$ be an effective listing of the requirements $\left\{P_{e}^{a}: e \in \omega\right.$ and $\left.a \in \bigcup_{x \in \omega} H_{x}\right\}$ and $\left\{Q_{e}^{a}: e \in \omega\right.$ and $\left.a \in \bigcup_{x \in \omega} M_{x}\right\}$. As in the proof of Theorem 1, we construct a recursive function $f: \omega \times \omega \rightarrow \omega$ and for $i \in \omega$ define $A_{i, s}=$ $\left\{\left\langle x, \alpha_{f\left(x, s^{\prime}\right)}(i)\right\rangle: x \in \omega\right.$ and $\left.s^{\prime} \leqslant s\right\}$, and $A_{i}=\bigcup_{s} A_{i, s}$. The proof of Theorem 1 is modified by first changing the definition of a legal move, and second changing the strategy for cooperation between coding and the $\left\{T_{i}: i \in \omega\right\}$.

We modify the legal move definition to get (5) and (6). Since the range of $f$ is now infinite, (5) and (6) are no longer automatic, but they will be if we establish

(39) There is a recursive function $F$ such that for $x, s \in \omega, f(x, s) \leqslant F(x)$.

As in Fejer and Shore [1], to establish (39) we define sets $S(i, y)$ for $i, y \in \omega$, where $S(i, y)$ represents $T_{i}$ 's guess at the possible values for $f(y, s)$. First we define 
$J\left(i, r_{1}, r_{2}\right)$ for $i, r_{1}, r_{2} \in \omega$ by

$$
J\left(i, r_{1}, r_{2}\right)= \begin{cases}\varnothing & \text { if } \exists a \in\left(\bigcup_{x \in \omega} H_{x}\right) \exists e \in \omega\left(T_{i}=P_{e}^{a}\right), \\ \varnothing & \text { if } \exists a \in\left(\bigcup_{x \in \omega} M_{x}\right) \exists e \in \omega\left(T_{i}=Q_{e}^{a}\right) \text { and } \\ & \alpha_{r_{1}}\left(k_{a}\right) \neq \alpha_{r_{2}}\left(k_{a}\right), \\ & \text { if } \exists a \in \bigcup_{x \in \omega} M_{x} \exists e \in \omega\left(T_{i}=Q_{e}^{a}\right) \text { and } \\ & \alpha_{r_{1}}\left(k_{a}\right)=\alpha_{r_{2}}\left(k_{a}\right), \text { and } s_{0}, \ldots, s_{m} \\ & \text { satisfy }(3) \text { for } p_{i_{a}} \wedge p_{j_{a}}=p_{k_{a}} \\ & \text { and } r_{1}, r_{2} .(\text { Choose one fixed } \\ & \text { set of interpolants for each } \\ & \text { such triple } \left.\left(a, r_{1}, r_{2}\right) .\right)\end{cases}
$$

We modify our recursive partition of $\omega$ so that we get

$$
\begin{aligned}
\left\{D(\langle e, a\rangle): e \in \omega, a \in \bigcup_{x \in \omega} H_{x}\right\} \cup\left\{W^{a}(x): x \in \omega, a \in H_{x}\right\} \\
\cup\left\{W^{(a, k)}(x): x \in \omega,(a, k) \in M_{x} \times\{1,2\}\right\} \cup\{C W(x): x \in \omega\}
\end{aligned}
$$

satisfying (15) and

Each $x \in \omega$ has two finite sets of numbers associated with it, call them $W(x)$ and $C W(x)$. Each element of $W(x) \cup C W(x)$ is greater than $x$, and $\min (C W(x))>\max (W(x))$. Partition

$$
\begin{aligned}
& W(x) \text { into sets } W^{a}(x) \text { for } a \in H_{x} \text { and } W^{(a, k)}(x) \text { for }(a, k) \in \\
& M_{x} \times\{1,2\} \text { so that }\left|W^{a}(x)\right|=2^{x+1} \text { for } a \in H_{x}, x \in \omega \text { and } \\
& \left|W^{(a, k)}(x)\right|=2^{x+1} \text { for }(a, k) \in M_{x} \times\{1,2\}, \quad x \in \omega \text { and } \\
& |C W(x)|=2 \cdot m_{x} \cdot 2^{x+1}\left(h_{x}+2 m_{x}\right) .
\end{aligned}
$$

As before, $g$ will be a 1-1 recursive function such that for all $x, g: W(x) \times M_{x} \times$ $\{1,2\} \rightarrow C W(x)$. For $a \in \cup_{x \in \omega} H_{x}, r(a)_{1}, r(a)_{2}$ are defined to satisfy (19), and for $(a, k) \in \bigcup_{x \in \omega} M_{x} \times\{1,2\}, r(a, k)_{1}, r(a, k)_{2}$ are defined to satisfy (20). We are now ready to define the $S(i, y)$. For $i \geqslant y+1$, define

$$
S(i, y)= \begin{cases}\left\{r(a), r(a)_{2}\right\} & \text { if } a \in \bigcup_{x \in \omega} H_{x} \text { and } \\ \left\{r(a, k)_{1}, r(a, k)_{2}\right\} & \text { if } \left.(a, k) \in \bigcup_{e \in \omega} D(\langle e, a\rangle)\right) \cup\left(\bigcup_{x \in \omega} W_{x} W^{a}(x)\right), \\ & \text { and } \exists z \in \bigcup_{x \in \omega} W(x), y=g(z, a, k), \\ \left\{r(a, k)_{1}, r(a, k)_{2}\right\} & \text { if }(a, k) \in \bigcup_{x \in \omega} M_{x} \times\{1,2\} \\ & \text { and } y \in \bigcup_{x \in \omega} W^{(a, k)}(x) .\end{cases}
$$


Given $S(i+1, y)$ with $0 \leqslant i \leqslant y$, define

$$
S(i, y)=S(i+1, y) \cup \bigcup\left\{J\left(i, r_{1}, r_{2}\right): r_{1}, r_{2} \in S(i+1, y)\right\} .
$$

We modify the definition of " $\sigma$ is a legal move for $T_{i}$ at $s+1$ " by replacing (22) with

$$
\begin{aligned}
& \text { For } x<|\sigma|(\sigma(x) \neq f(x, s) \rightarrow x>R(i, s) \text { and } x>i \text { and } \\
& f(x, s)=f(x, 0) \text { and } \sigma(x) \in S(i, x)) .
\end{aligned}
$$

Now for all $y$, if for some $s, f(y, s+1) \neq f(y, 0)$, then this definition of a legal move ensures that $f(y, s+1) \in S(0, y)$. Hence if we define $F(x)=\max (S(0, x))$, then the new construction will satisfy (39). The proof of the analog of Lemma 6 for the new construction remains basically the same as the original proof. When considering $T_{i}=Q_{e}^{a}$, the legal move $\mu$ at stage $s_{2}$ constructed in the proof is such that if $\mu(y) \neq f\left(y, s_{2}\right)$, then $y=g\left(z, a^{\prime}, k^{\prime}\right)$ for some $z, a^{\prime}, k^{\prime}$, or $\exists s^{\prime} \geqslant s_{2}$, $f\left(y, s^{\prime}+1\right) \neq f\left(y, s^{\prime}\right)$. If $y=g\left(z, a^{\prime}, k^{\prime}\right)$, then $\mu(y)=r\left(a^{\prime}, k^{\prime}\right)_{2} \in S(i, y)$. If $\exists s^{\prime} \geqslant$ $s_{2}\left(f\left(y, s^{\prime}+1\right) \neq f\left(y, s^{\prime}\right)\right)$, then some $T_{j}, j>i$, was active at $s^{\prime}+1$, and $f\left(y, s^{\prime}\right)$, $f\left(y, s^{\prime}+1\right) \in S(j, y) \subseteq S(i+1, y)$. We constructed $\mu$ so that $\mu(y)=$ one of the interpolants for $\alpha_{f\left(y, s^{\prime}\right)}\left(k_{a}\right)=\alpha_{f\left(y, s^{\prime}+1\right)}\left(k_{a}\right)$, and so $\mu(y) \in S(i, y)$. Hence the $\mu$ constructed will satisfy the new definition of legal move, and the proof of Lemma 6 is as before.

The coding strategy is modified as follows. The list of relations $\left\{R_{a}: a \in \omega\right\}$ is infinite now, and so our coding action for $x$ cannot cooperate with all of the $R_{a}$. Instead we insist that the action for coding $x$ cooperate with $R_{a}$, only for $a<x$. Thus our action for coding the fact " $x \in K$ " is chosen from among $h_{x}+3 m_{x}$ many possibilities, and we still get a tt-reduction for $K$ from $A_{1}$. We establish

$$
\begin{aligned}
& \forall x \in \omega\left(x \in K \leftrightarrow \exists a \in H _ { x } \exists y \in W ^ { a } ( x ) \left(\left\langle y, \alpha_{r(a)_{2}}\right\rangle \in A_{1}\right.\right. \text { and } \\
& \forall\left(a^{\prime}, k^{\prime}\right) \in M_{x} \times\{1,2\} \forall r \leqslant F\left(g\left(y, a^{\prime}, k^{\prime}\right)\right) \\
& \left.\left.\left\langle r \neq r\left(a^{\prime}, k^{\prime}\right)_{1} \rightarrow\left\langle g\left(y, a^{\prime}, k^{\prime}\right), \alpha_{r}(1)\right\rangle \notin A_{1}\right\rangle\right)\right)
\end{aligned}
$$

or

$$
\begin{aligned}
& \exists(a, k) \in M_{x} \times\{1,2\} \exists y \in W^{(a, k)}(x) \\
& \left\langle y, \alpha_{r(a, k)_{2}}\right\rangle \in A_{1} \text { and } \forall\left(a^{\prime}, k^{\prime}\right) \in M_{x} \times\{1,2\} \\
& \forall r \leqslant F\left(g\left(y, a^{\prime}, k^{\prime}\right)\right)\left(r \neq r\left(a^{\prime}, k^{\prime}\right)_{1} \rightarrow\left\langle g\left(y, a^{\prime}, k^{\prime}\right), \alpha_{r}(1)\right\rangle \notin A_{1}\right) .
\end{aligned}
$$

The proofs of the lemmas showing the correctness of the construction will be as before, except that when considering $T_{i}=Q_{e}^{a}$ or $P_{e}^{a}$, we start from a stage $s_{0}$ such that $K_{s_{0}} \uparrow a=K \uparrow a$; then for $s \geqslant s_{0}$, if $T_{i}$ has priority at $s$, then $T_{i}$ will not be injured by coding at $s$. Thus Lemmas $3,4,5$ and 6 will hold if we replace $[1, h]$ by $H_{x}$ or $\bigcup_{x \in \omega} H_{x},[h+1, m]$ by $M_{x}$ or $\bigcup_{x \in \omega} M_{x}$ whenever appropriate and let $s_{0}$ be large enough so that $K_{s_{0}} \uparrow a=K \uparrow a$.

COROLlaRY 2. The $\exists$ theory of the r.e. tt-degrees in the language $\mathscr{L}$ with $\leqslant, \vee, \wedge$ as relation symbols and 0,1 as constant symbols is decidable.

Proof. The decision procedure is the same as the one used in Fejer and Shore [1]. An existential sentence $\sigma=\exists x_{1}, \ldots, \exists x_{n} \varphi$ in $\mathscr{L}$ will be valid in the r.e. $t$-degrees iff it is valid in some partial order with distinct least and greatest elements and at most 
$(n+2)+2(n+2)^{3}$ elements. Suppose $\sigma$ is valid in some finite partial order $\mathscr{P}$ with distinct least and greatest elements. $\mathscr{P}$ can be extended to a finite lattice, $\mathscr{P}^{\prime}$, with distinct least and greatest elements. Since $\mathscr{P}^{\prime}$ is finite, it is recursively presentable, and so by Theorem $2 \mathscr{P}^{\prime}$ can be embedded in the r.e. tt-degrees. The embedding preserves all joins and infs mentioned in $\varphi$, as well as least and greatest elements, and so $\sigma$ will be valid in the r.e. tt-degrees. Next suppose that $\sigma$ is valid in the r.e. tt-degrees and that $\mathbf{a}_{1}, \ldots, \mathbf{a}_{n}$ is a sequence satisfying $\varphi$. We construct a partial order $\mathscr{P}$ containing $\mathbf{a}_{1}, \ldots, \mathbf{a}_{n}, \mathbf{0}, \mathbf{1}$ and enough other r.e. tt-degrees so that if $\mathbf{b}, \mathbf{c}, \mathbf{d} \in$ $\left\{\mathbf{a}_{1}, \ldots, \mathbf{a}_{n}, \mathbf{0}, \mathbf{1}\right\}$ and $\mathbf{b}, \mathbf{c} \leqslant \mathbf{d}$, and $\mathbf{b} \vee \mathbf{c} \neq \mathbf{d}$, then $\mathscr{P}$ contains an $\mathbf{e}$ so that $\mathbf{b}, \mathbf{c} \leqslant \mathbf{e}$ and $\mathbf{e}<\mathbf{d}$. Likewise if $\mathbf{d} \leqslant \mathbf{b}, \mathbf{c}$ and $\mathbf{b} \wedge \mathbf{c} \neq \mathbf{d}$ then $\mathscr{P}$ contains an $\mathbf{e}$ so that $\mathbf{e} \leqslant \mathbf{b}, \mathbf{c}$ and $\mathbf{d}<$ e. $\mathscr{P}$ will have no more than $(n+2)+2(n+2)^{3}$ elements, and $\sigma$ will be valid in $\mathscr{P}$.

\section{REFERENCES}

1. P. A. Fejer and R. A. Shore, Embeddings and extension of embeddings in r.e. degrees for strong reducibilities, Proc. Oberwolfach "Recursion Theoretic Week 1984" (H. D. Ebbinghaus, G. H. Muller and G. E. Sacks, eds.), Springer-Verlag, Berlin, 1985.

2. C. A. Haught, Turing and truth table degrees of 1-generic and recursively enumerable sets, Dissertation, Cornell Univ., Ithaca, N. Y., 1985.

3. C. G. Jockusch, Jr. and J. Mohrherr, Embedding the diamond lattice in the recursively enumerable truth table degrees, Proc. Amer. Math. Soc. 94 (1985), 123-128.

4. P. G. Odifreddi, Strong reducibilities, Bull. Amer. Math. Soc. (N.S.) 4 (1981), 37-86.

5. P. Pudlak and J. Tuma, Every finite lattice can be embedded in a finite partition lattice, Algebra Universalis 10 (1980), 74-95.

6. H. Rogers, Theory of recursive functions and effective computability, McGraw-Hill, New York, 1967.

7. R. A. Shore, Finitely generated codings and the degrees r.e. in a degree d, Proc. Amer. Math. Soc. 84 (1982), 256-263.

Department of Mathematical Sciences, Loyola University of Chicago, Chicago, Illinois 60626 